UNIVERSITY OF KWAZULU-NATAL

College of Agriculture, Engineering and Science

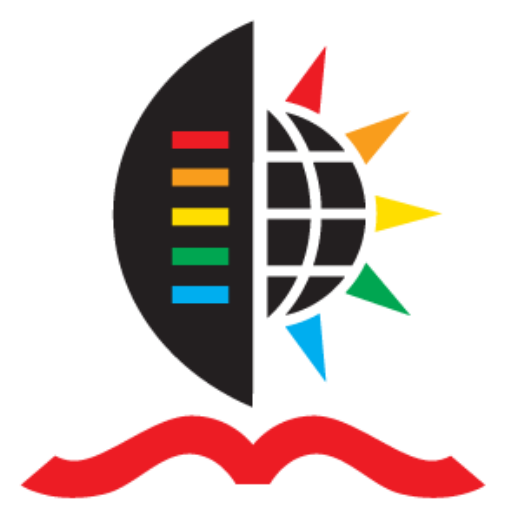

\title{
Unsupervised Caries Detection in Non-Standardized Bitewing Dental X-Rays by
}

Darren John Osterloh

Supervisor:

Prof. Serestina Viriri

A dissertation submitted in fulfillment of the academic requirements for the degree of Master of Science in Computer Science

in the

School of Mathematics, Statistics and Computer Science

March 2017 


\section{Declaration of Authorship}

I, Darren John Osterloh, declare that this thesis titled, 'Unsupervised Caries Detection in Non-Standardized Bitewing Dental X-Rays' and the work presented in it are my own. I confirm that:

- This work was done wholly or mainly while in candidature for a research degree at this University.

- Where any part of this thesis has previously been submitted for a degree or any other qualification at this University or any other institution, this has been clearly stated.

- Where I have consulted the published work of others, this is always clearly attributed.

- Where I have quoted from the work of others, the source is always given. With the exception of such quotations, this thesis is entirely my own work.

- I have acknowledged all main sources of help.

- Where the thesis is based on work done by myself jointly with others, I have made clear exactly what was done by others and what I have contributed myself.

\section{Darren John Osterloh}




\section{Declaration - Supervisor}

As the candidate's supervisor, I agree to the submission of this dissertation

Prof. Serestina Viriri 


\section{Declaration - Publications}

DETAILS OF CONTRIBUTION TO PUBLICATIONS that form part and/or include research presented in this dissertation

Publication 1:

Darren Osterloh, Serestina Viriri. Unsupervised Caries Detection in Non-standardized Bitewing Dental X-Rays. Lecture Notes in Computer Science (Springer), vol. 10072, pages 1-10, 2016.

Darren John Osterloh 


\begin{abstract}
In recent years dental image processing has become a useful tool in aiding healthcare professionals diagnose patients by reducing some of the problems inherent with dental radiographs. Despite advances in the field, accurate diagnoses of dental caries using Comptuer-aided Diagnosis (CAD) tools are still problematic due to the non-uniform nature of dental X-rays. The reason as to why accurate diagnoses are problematic is in part due to exisiting systems utilizing a supervised learning model for their diagnostic algorithms. Using this approach results in a detection system which is trained to identify caries under specific conditions. When the input images vary greatly from the training set, these systems have a tendency to misdiagnose patients or miss possible caries altogether.

A method for the segmentation of teeth in periapical X-Rays is presented in this dissertation as well as a method for the detection of caries across a variety of non-uniform X-ray images using an unsupervised learning model. The diagnostic method proposed in this dissertation uses an assessment protocol similar to how dentists evaluate the presence of caries. Using this assessment protocol results in caries being evaluated relative to the image itself and not evaluated relative to a set of identifiers obtained from a learning model. The viability of an unsupervised learning model, and its relative effectiveness of accurately diagnosing dental caries when compared to current systems, is indicated by the results detailed in this dissertation. The proposed model achieved a $96 \%$ correct diagnostic which proved competitive with existing models.
\end{abstract}




\section{Acknowledgements}

I would like to thank Prof. Serestina Viriri, who acted as my supervisor and mentor throughout the duration of my Master's degree. Without his guidance and support this dissertation would not have been possible.

A special thanks also goes to the Dental Clinic at the Universiti Teknologi Malaysia (UTM), who provided the dental X-rays that were used to complete this dissertation. 


\section{Contents}

Declaration of Authorship i

Declaration - Supervisor $\quad$ ii

$\begin{array}{ll}\text { Declaration - Publications } & \text { iii }\end{array}$

Abstract iv

Acknowledgements $\quad$ v

$\begin{array}{ll}\text { List of Figures } & \text { ix }\end{array}$

List of Tables $\quad$ xi

Abbreviations $\quad$ xii

1 Introduction 1

1.1 Motivation ...................... 4

1.2 Problem Statement . . . . . . . . . . . . . . . . . . 5

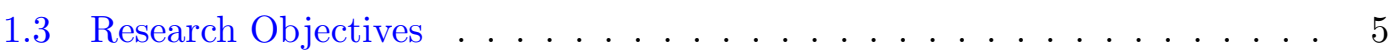

1.4 Contributions of the Dissertation . . . . . . . . . . . . . 6

1.5 Organization of the Dissertation $\ldots \ldots \ldots \ldots$

2 Background and Related Work $\quad 9$

2.1 Introduction . . . . . . . . . . . . . . . . . . . . 9

2.2 Anatomy of a tooth . . . . . . . . . . . . . . . . 9

2.3 Dental Radiography for Caries Detection . . . . . . . . . . . . . . . . 11

2.4 Related Work . . . . . . . . . . . . . . . . . . . . . . . 11

2.4 CAD Systems . . . . . . . . . . . . . . . . 11

2.4 .2 Enhancement Techniques . . . . . . . . . . . . . . 12

2.4.3 Segmentation Methods . . . . . . . . . . . . . . . . . . 12

2.4.4 Caries Detection Methods . . . . . . . . . . . . . . . . . . 14

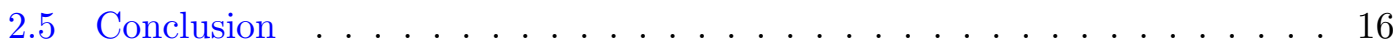

$\begin{array}{llr}3 & \text { Teeth Segmentation } & 17\end{array}$ 
3.1 Introduction . . . . . . . . . . . . . . . . . . . . . . 17

3.2 Pre-Processing . . . . . . . . . . . . . . . . . . . . . . . . 18

3.2 .1 Image Cleanup . . . . . . . . . . . . . . . . . . . . . . 18

3.2 .2 Image Enhancement . . . . . . . . . . . . . . . . . . . . . 19

3.3 Thresholding . . . . . . . . . . . . . . . . . . 20

3.3 .1 Iterative Thresholding . . . . . . . . . . . . . . . 20

3.3.2 Adaptive Thresholding . . . . . . . . . . . . . . . . . . . . . . . . . . . 21

3.4 Tooth Separation . . . . . . . . . . . . . . . . . . . . 22

3.4 .1 Integral Projection . . . . . . . . . . . . . . . . 22

3.4 Line Selection . . . . . . . . . . . . . . . . . . . . 24

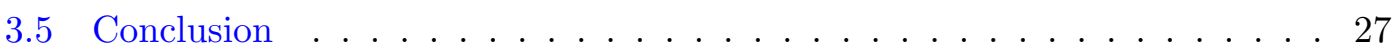

4 Tooth Boundary Detection $\quad \mathbf{2 8}$

4.1 Introduction . . . . . . . . . . . . . . . . . 28

4.2 Top and Bottom Hat Transformations . . . . . . . . . . . . . . 29

4.3 Boundary Detection . . . . . . . . . . . . . . . . . . . 30

4.4 Search Space Refinement . . . . . . . . . . . . . . . . . . . . 32

4.4 Centre Elimination . . . . . . . . . . . . . . . . . . 32

4.4 Boundary Dilation . . . . . . . . . . . . . . . . . . . . . . . . . . . . . . 33

4.5 Conclusion . . . . . . . . . . . . . . . . . . 34

5 Dental Caries Detection $\quad \mathbf{3 6}$

5.1 Introduction . . . . . . . . . . . . . . . . . . 36

5.2 Blob Detection . . . . . . . . . . . . . . . . 36

5.3 Caries Analysis . . . . . . . . . . . . . . . . . . . . . . . . . . . . . . . . . . . . .

5.3 .1 Region of Interest Generation . . . . . . . . . . . . . . . . . . . . . . . . . . . . .

5.3 .2 Cluster Analysis . . . . . . . . . . . . . . . . . . . . . . . . . . . . . . . . . . . . . . . .

5.4 Conclusion . . . . . . . . . . . . . . . . . . 41

6 Results and Discussion $\quad 42$

6.1 Introduction . . . . . . . . . . . . . . . . . . . . . . . . 42

6.2 Dataset . . . . . . . . . . . . . . . . . . . 43

6.2.1 X-Ray Errors and Artifacts . . . . . . . . . . . . . . 43

6.2.1.1 Missing Crown . . . . . . . . . . . . . . 43

6.2.1.2 PID Alignment Artifact . . . . . . . . . . . . . . 44

6.2.1.3 Exposure Errors . . . . . . . . . . . . . . . . . . 44

6.2 .2 The Dataset. . . . . . . . . . . . . . . . . . 45

6.3 Caries Detection Framework . . . . . . . . . . . . . . . . . 46

6.4 Segmentation Results . . . . . . . . . . . . . . . . . . . . . . . . . 48

6.5 Caries Detection Results . . . . . . . . . . . . . . . . . . 50

6.6 Conclusion . . . . . . . . . . . . . . . . . . 52

7 Conclusion and Future Work $\quad 53$

7.1 Conclusion ........................... 53

7.2 Future Work . . . . . . . . . . . . . . . . . 55 
Bibliography 


\section{List of Figures}

1.1 A flow diagram of a generic CAD System . . . . . . . . . . . . 4

2.1 Anatomy of a tooth showing cross section of a molar tooth with gum and jawbone $[1] \ldots \ldots \ldots \ldots 10 \ldots \ldots \ldots$

3.1 An example of the image cleanup process. (a) shows the original image. (b) shows the image after artifact and reference information bar removal . 18

3.2 An example of the image enhancement process. (a) shows the original image. (b) shows the image after the median filter and histogram equalization have been applied. (c) shows the effects of the histogram equalization process . . . . . . . . . . . . . . . . . . . 19

3.3 An example showing the results of the different approaches used to obtain a global mask threshold from adaptive thresholding. (a) shows the original image. (b) shows the result of generating a threshold from the global adaptive values. (c) shows the result of generating a threshold value from the image itself and $(\mathrm{d})$ shows the result of the hybrid approach . . . . . . 22

3.4 An example of vertical integral projection detecting the gaps between the mask image of the X-ray . . . . . . . . . . . . . . . . . . . . 23

3.5 Examples of where valley detection incorrectly identified the crest between molar roots as a gap . . . . . . . . . . . . . . . . 23

3.6 An example of where the separation line attempts to bybass the gap between teeth in favour of a line with fewer intersection points . . . . . . 26

3.7 Examples of how the crest detection algorithm correctly removed the separation lines for the images in $3.5 \ldots \ldots 26$

4.1 Examples of the top and bottom hat image transformations. (a) shows the images after their separation in the previous chapter. (b) shows the images after the transformation . . . . . . . . . . . . . 30

4.2 Examples of image degradation due to image transformations and binary thresholding. (a) shows the images after their top and bottom hat transformations. (b) shows the images after the binary threshold . . . . . . . 31

4.3 Examples of boundary detection using active contours. (a) shows images of molar teeth where the curvature of the boundary around the root is not perfect. (b) shows images of boundaries detected on other teeth . . . 32

4.4 Examples of the centre elimination method. (a) shows examples of where this method correctly identifies the centre region of each tooth. (b) shows examples of where the centre region is defined incorrectly or results in known caries to be excluded . . . . . . . . . . . . . . . . . 33

4.5 Examples of the boundary dilation method for search space refinement. . 34 
5.1 Examples of images displaying potential caries sites highlighted by the blob detector . . . . . . . . . . . . . . . . . . . 37

5.2 The two types of connectivity kernels which can be used for connectivity

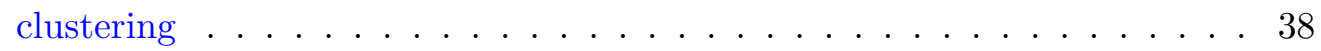

5.3 Examples of the caries clusters obtained from the connectivity cluster kernel (a) shows the clusters on the X-rays. (b) shows the isolated clusters 38

6.1 Example of dental X-ray with missing crown . . . . . . . . . . . . 43

6.2 Example of a PID alignment artifact . . . . . . . . . . . . . . . . 44

6.3 An example of the different exposure rate errors. (a) shows an image with an underexposed film. (b) shows an image with an overexposed film . . . 44

6.4 Examples of the different types of X-rays in the dataset. (a). shows an Xray with an impacted wisdom tooth. (b). shows an X-ray with a missing tooth. (c). shows an X-ray where the contrast darkens across the image. . 45

6.5 Overview of the caries detection framework. . . . . . . . . . . . 47

6.6 Examples of segmentation results across the dataset . . . . . . . . . . . . 49

6.7 Examples of the results obtained by the detection algorithm and the comparison with the ground truth. (a). the original image. (b). the segmented image with detected caries. (c). the ground truth data . . . . 51 


\section{List of Tables}

6.1 Dataset . . . . . . . . . . . . . . . . . . 45

6.2 Segmentation Results . . . . . . . . . . . . . . . . . . . . . . . 48

6.3 Region Specific Segmentation Results Comparison . . . . . . . . . . . . . . 48

6.4 Overall Segmentation Results Comparison . . . . . . . . . . . . . . . . . 49

6.5 Caries Identification Results . . . . . . . . . . . . . . . . . . 50

6.6 Caries Identification Results Breakdown . . . . . . . . . . . . . . . . 50

6.7 Caries Identification Results Comparison . . . . . . . . . . . . . . . . 51 


\title{
Abbreviations
}

\author{
G. V. Greene Vardiman \\ CAD Computer-Aided Diagnosis \\ AHE Adaptive Histogram Equalization \\ CLAHE Contrast Adaptive Histogram Equalization \\ MAHE Median Adaptive Histogram Equalization \\ SCLAHE Sharp Contrast Adaptive Histogram Equalization \\ FIR Finite Impulse Response \\ CT Computerized Tomography \\ PID Position Indicating Device
}




\section{Chapter 1}

\section{Introduction}

Dental caries are one of the most prevalent diseases present in modern society, with approximately $36 \%$ of the world's population showing symptoms of caries [2]. In the past, developing countries were considered to be less at risk due to their lower consumption of sugary foods. Africa in particular had a $19 \%$ lower rate of infection compared to the world average, whilst the American and European regions had a $14 \%$ and $10 \%$ higher infection rate respectively when compared to the average [3]. Despite this previously low infection rate, changing living conditions have increased the rate of tooth decay in African countries thus worsening the already prevalent disease. In order to keep the disease under control it is important to treat it during its early stages, before advanced tooth decay occurs.

Dental caries, also known as cavities, refers to the bacterial breakdown of the hard tissues of the tooth [4]. The occurrence of caries is the result of dental plaque forming bacteria releasing acid in the presence of fermentable carbohydrates. Glucose, fructose and sucrose produce this reaction [5] thus a diet high in simple sugars accelerates this process. The acid released during this process causes de-mineralization of the surface of the tooth. Dental caries occur when the rate of mineral build up is less than the rate of decay. Dental caries are typically categorized using Greene Vardiman (G. V.) Black Caries Classification [6]. This protocol categorizes the carious lesion based on the tooth affected, as well as the location of the lesion. These different categories are defined as follows: 
- Class I Refers to caries on the occlusal surfaces of posterior teeth, buccal or lingual pits on molars or lingual pit near cingulum of maxillary incisors

- Class II Refers to caries on the proximal surfaces of posterior teeth

- Class III Refers to caries on the interproximal surfaces of anterior teeth without incisal edge involvement

- Class IV Refers to caries on the interproximal surfaces of anterior teeth with incisal edge involvement

- Class V Refers to caries on the cervical third of the facial or lingual surface of the tooth

- Class VI Refers to caries on the incisal or occlusal edge worn away due to attrition

In addition to this positional classification, caries are further categorized based on the severity of their progress. This is based on the amount of enamel and dentin which has been affected by the caries lesion [7]. Incipient lesions are those which have a depth of less than half the enamel. Moderate lesions penetrate more than halfway through the enamel but do not affect the dentin. Advanced lesions either extend to the dentin region or penetrate it but do not extend more than halfway through. In severe caries lesions, the lesion extends more than halfway through the dentin region and may even reach the pulp of the tooth.

Caries falling under classes I, IV and VI can be identified during a clinical inspection as they lie on regions of the tooth which are visible to the naked eye. Caries which are categorized into classes II, III and V fall on the surfaces between teeth and as a result can only be viewed by X-rays.

The discovery of the X-ray allowed for great advancements in the field of dentistry. Prior to its introduction to the medical field, diagnoses could only be made from a visual inspection of a patient's teeth. With the use of X-rays, oral healthcare professionals were able to view previously unobservable regions thus allowing them to detect caries which would have gone untreated until visible decay was present [8]. The ability to diagnose dental caries in this manner proved to be greatly beneficial and is still used to this day. 
Depending on the form of treatment that is required, varying degrees of information are needed. To accommodate this, there are three types of X-rays commonly used to diagnose dental health [9].

- Bitewing X-rays are used to obtain crown images of the back teeth. These images show the upper and lower molars as well as the bicuspids. These X-rays are used to detect if decay is present between the back teeth and also indicate if there is a problem with tooth alignment.

- Periapical X-rays only display one or two teeth in a single image. Similarly to bitewing X-rays, periapical X-rays also show the crown of the tooth, however they extend the entire length of the tooth revealing the condition below the gum line. These X-rays are used to detect tooth decay as well as other problems below the gum line such as impacted teeth, abscesses, cysts and tumours. They also display information relating to tooth bone height and root tips.

- Panoramic X-rays give a complete view of the dental region, displaying all upper and lower teeth as well as the sinuses and jaw joints. Unlike the previous two types of X-rays, panoramic X-rays require a specialized machine in order to pan the Xray emitting tube around the back of the patient's head in order to capture the whole image on a single film. This type of X-ray has the same diagnostic capability as a periapical X-ray and as such is used to identify abscesses, cysts, caries, tumors or any irregularities with the structure of the jaw region itself.

Dental X-rays are typically noisy and low in contrast due to the low dosage rates in the capture process [10]. These low dosage rates can also affect the visibility of caries due to the X-rays not fully penetrating the teeth. Low dosage rates are used to ensure the health of the patient [11]. Whilst it is important to adhere to these low dosage rates, it does lead to the necessity of image enhancement techniques in order to maintain diagnostic accuracy by enhancing the contrast of the image and reducing the overall noise levels [12].

In recent years image processing has become an invaluable tool in the medical field. Computer-Aided Diagnosis (CAD) systems have become a vital tool in medical radiology, being used extensively in the diagnosis of lung nodules and breast cancer [13]. 


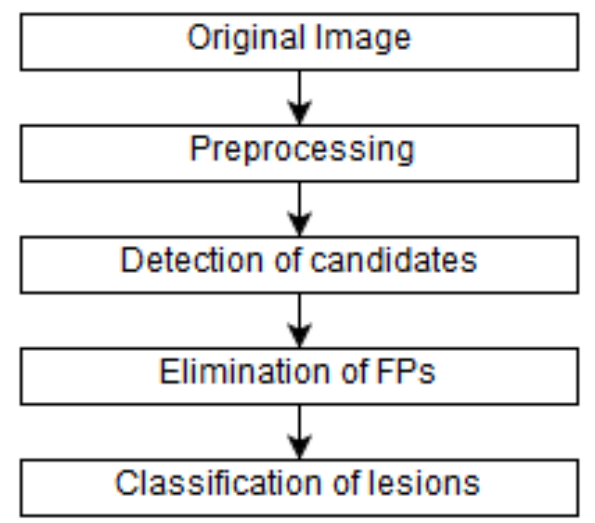

Figure 1.1: A flow diagram of a generic CAD System

Research into the use of CAD systems for caries diagnosis has shown that correct caries identification rates improve when compared to non-assisted assessments. Figure 1.1 outlines the generic CAD system used for bio-medical diagnostics as described by [14]. The process begins with the preprocessing of input images which includes image enhancement and segmentation. The next stage is the detection of possibe candidates which, for the purpose of this dissertation, will refer to caries lesions. Following the detection of possible caries, the CAD system attempts to reduce the presence of false positives detected from the previous stage. Finally the system classifies the lesions using a diagnostic algorithm.

Due to CAD systems offering an increased detection rate of caries in affected patients, healthcare professionals have started to adopt them as part of the diagnostic process. Despite the recorded benefit of using these systems, the adoption rate has been sluggish, mostly due to existing systems needing to be used in tandem in order to make up for their individual shortcomings [15].

\subsection{Motivation}

The goal of this research work is to obtain a method for caries identification that uses an unsupervised learning model. Current models dealing with dental X-rays predominately focus on tooth segmentation, identification of individuals through dental profile matching, or the identification of caries using a supervised learning model. In the case of the latter, feature extraction is done for the purpose of comparing the results against a database of previously extracted features. An assessment is then made based on the 
suspected caries' similarity to previously encountered caries. By combining existing methods and adapting them accordingly it is possible to develop a new analytical model which is able to process X-rays without a comparative database. The combination of these methods leads towards a novel caries detection approach with a diagnostic potential that is not influenced by the conditions unique to the learning set.

\subsection{Problem Statement}

There is limited research with respect to caries identification in literature. Existing methods all follow a similar protocol of boundary detection using active contours followed by feature extraction along said boundary. In the case of supervised learning models, the statistical, region based, border and region texture [16] are collected and compared against existing data. If any similarities are detected in this comparison the tooth is flagged for caries. As a result, supervised learning models have high sensitivity (few false negatives) and low specificity (many false positives) [17][18].

An unsupervised learning model uses an image analysis approach to diagnose the presence of dental caries. An assessment of the area surrounding a potential caries region is done to determine if caries are present. This model has the advantage of faster diagnostic rates as it does not need to iterate through an entire database in order to obtain a diagnosis. In addition, it has the added advantage of reducing the rate of false positives, as supervised assessment models make positive predictions if only a portion of caries hallmarks are present. This leads to false positives if the analyzed image has characteristics similar to caries in the training set which do not represent caries in the new image.

\subsection{Research Objectives}

The primary objective of this research is to model a framework for a novel caries identification method using an unsupervised learning approach. This proposed framework uses the analytical techniques utilized by dental healthcare professionals when assessing the presence of dental caries, with the image enhancement techniques used by current 
systems prior to feature extraction. A segmentation model which preserves the boundary details of each tooth is also required in order to obtain accurate results. The overall goal of this dissertation is to ensure the model proposed is of comparative or better diagnostic ability to those already implemented. The specific objectives which must be met break down as follows:

- To accurately segment teeth in dental X-rays using different segmentation methods

- To model an unsupervised framework for caries identification

\subsection{Contributions of the Dissertation}

The main contributions of this research are the proposal of a novel segmentation method and a novel caries detection method based on image analysis protocols. These contributions are described as follows:

\section{- A segmentation method using rotational and probabilistic acceptance:}

The segmentation method determines the line of best separation by utilizing a weighted algorithm of the two algorithms. Traditional segmentation methods determine if a line is the best candidate for separation by using only one of the acceptance algorithms. Rotational acceptance is implemented when the image only contains tooth structures and the best line can be equated to the one which hits the least number of points. The probabilistic acceptance algorithm is used when the tooth distribution is predictable and the position of the next tooth gap can be determined from the position of the previous one. In situations where neither of these cases are present, existing acceptance protocols lead to incorrectly segmented images. By using a weighted combination of the two algorithms, which are adapted to provide accurate results in all X-ray types, X-ray images can be accurately segmented whilst preserving details crucial to caries detection.

- A caries detection framework based on image analysis protocols: The caries detection framework is applied to the images obtained from the application of the segmentation method. The proposed method involves three steps for determining the caries region and diagnosing it. The boundary detection step adapts 
the work of Oliveira [16] who required boundary details for a supervised learning approach. It utilizes top and bottom hat transformations to remove unwanted structures and assist with active contours. The second step involves the detection of potential caries regions by first using a blob detector to generate a rough region of interest, and then applying a connectivity clustering model to isolate the potential caries location. Finally, the last step uses sobel operators and gradient analysis to determine if the region of interest does contain caries.

\subsection{Organization of the Dissertation}

The remainder of the dissertation is organized as follows:

- Chapter 2 - Background and Related Work The first part of this chapter looks at teeth and how practical identification of caries takes place. The remainder of the chapter looks at existing research in the field of dental X-Rays.

- Chapter 3 - Teeth Segmentation This chapter covers the first stage of the caries identification method and mostly covers how existing methods are adapted to best suit this dissertation. The first part of this chapter looks at image enhancement techniques used to optimize segmentation. The second part of this chapter looks at thresholding techniques used to isolate the teeth in the X-rays from their background pixels. The final part of this chapter looks at the integral projection method used as well as the selection algorithms used to obtain the best segmentation lines.

- Chapter 4 - Tooth Boundary Detection This chapter covers the second stage of the caries identification method. This chapter covers the use of top and bottom hat transformations in order to enhance image contrast, the use of active contours to obtain the edge boundary of each tooth and looks at two different methods to isolate the search space for caries detection.

- Chapter 5 - Dental Caries Detection This chapter covers the final stage of the caries identification method. The details of the methods used to isolate potential caries and evaluate their validity are looked at. 
- Chapter 6 - Results and Discussion This chapter covers the results of the various methods implemented in the previous chapters as well as a detailed overview of the dataset used for testing.

- Chapter 7 - Conclusion and Future Work This chapter concludes the dissertation and discusses future work. 


\section{Chapter 2}

\section{Background and Related Work}

\section{$2.1 \quad$ Introduction}

This research work focuses on the identification of caries in dental X-rays through a non-supervised learning model. This problem is unique as the majority of diagnostic algorithms do not implement image analysis as a means of diagnosis, rather opting to use a comparative assessment model to compare the similarities of potential caries regions to those previously encountered. Using these algorithms results in either high false positive or false negative rates depending on the system's inability to handle deviation from the source data. In order to achieve a more reliable analysis model, an image analysis approach is proposed. In this chapter, the anatomy of the tooth is introduced, current work regarding image enhancement and tooth segmentation is discussed and the various methods with which caries are isolated for analysis are presented.

\subsection{Anatomy of a tooth}

The tooth is a small whitish structure found in the jaw of humans and other animals. The number of teeth present ranges from 20 primary (milk) teeth in children to 28-32 permanent teeth in adults [19]. The variance is due to the mandibular third molars (wisdom teeth) which may not form in some of the population and in some cases where formation does occur, may not erupt from the gum line. 
The tooth is broken down into three main regions, the crown, which is the only part of the tooth visible above the gum line, the neck, which connects the crown region to the root, and the root, the part of the tooth that sits inside the bone socket [20].

Each tooth is composed primarily of hard tissues which protect the soft tissue in the centre. The outermost layer of the tooth is made of enamel which is the hardest tissue in the human body. Beneath this layer is the dentin which acts as a secondary defense. If caries are able to penetrate the enamel and reach this region the rate of decay increases as the dentin is softer thus resulting in faster decay [21]. At the centre of the tooth is the dental pulp which houses the nerve tissue and blood vessels for the tooth. Figure 2.1 shows in detail the cross-section of a tooth as described.

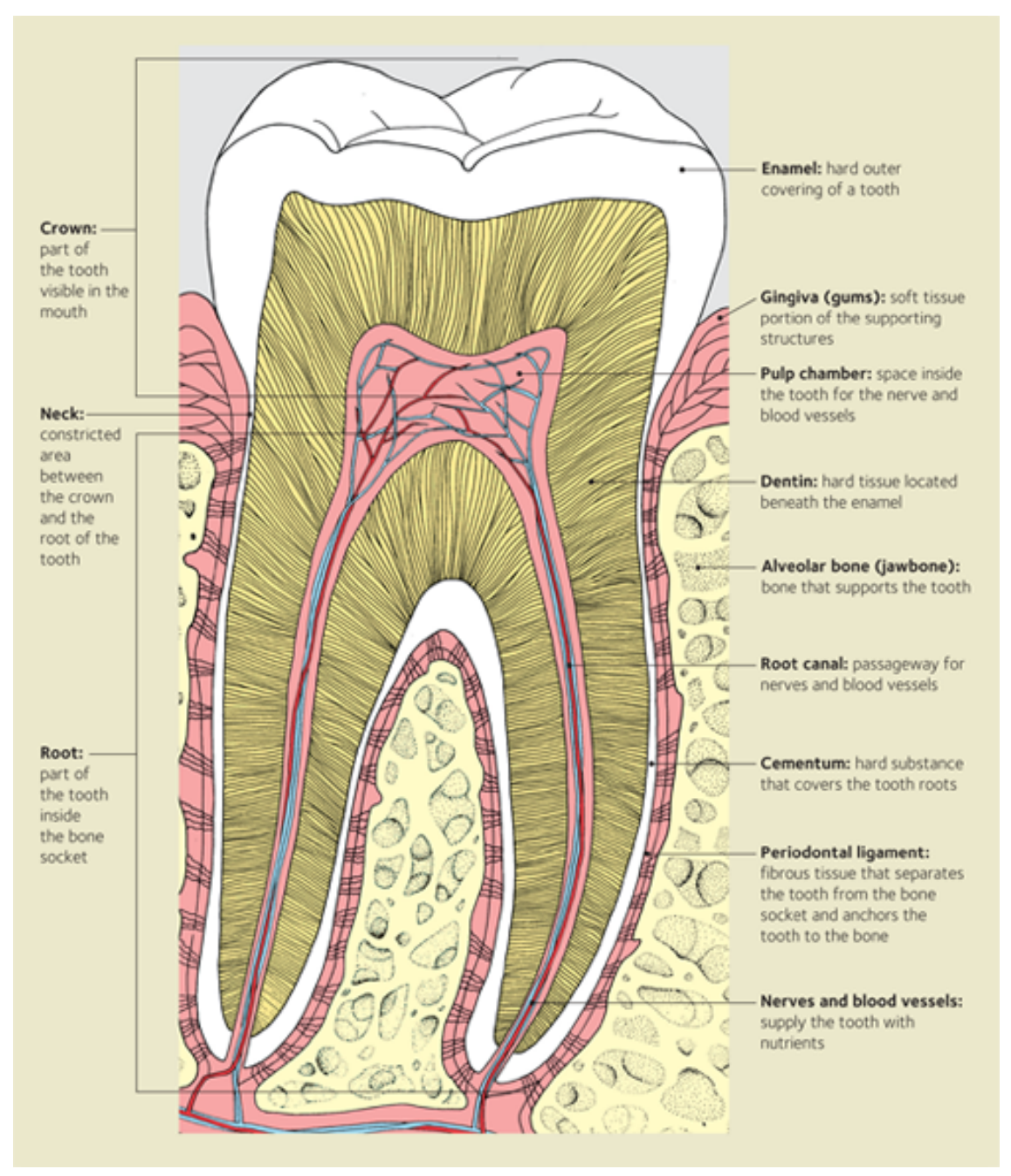

FiguRE 2.1: Anatomy of a tooth showing cross section of a molar tooth with gum and jawbone [1]. 


\subsection{Dental Radiography for Caries Detection}

Oral healthcare professionals have a variety of diagnostic methods at their disposal which they can use to diagnose dental caries in patients. In the past dentists used a dental explorer to probe the surface of a patient's teeth in order to determine whether caries were present. This practise has since died down due to the high misdiagnosis rate and the potential to aggravate early stages of decay [22]. Fluorescent methods, whereby a fluorescent light or laser is used to illuminate a tooth, have proven to have a higher diagnostic accuracy when detecting proximal caries [23]. For inter proximal caries, X-ray based imaging still proves to be the most effective diagnostic tool available [23].

X-rays penetrate the teeth at varying degrees, based on anatomical density. Fillings and teeth appear white to pale gray in X-rays as less radiation is able to penetrate, whilst areas with dental caries appear a darker shade of gray due to their impact on the surrounding bone structure. Gaps between teeth appear near black when correct X-ray standards are followed. Dental caries appear as darker regions along the boundary of the tooth, with severe infections penetrating deeper into the enamel and dentin.

\subsection{Related Work}

\subsubsection{CAD Systems}

In a study conducted by Tracy et al. [24], when presented with a collection of radiographs consisting of both restored and non-restored surfaces, dentists were able to correctly identify $30 \%$ of the caries present in the images. When presented with enhanced images the diagnosis rate increased to $39 \%$. With the aid of CAD systems the success rate increased to $69 \%$. Dykstra [25] noted similar findings were obtained from a study conducted at the University of California School of Dentistry, where the misdiagnosis rate of caries when they were present was $40 \%$. Furthermore, healthy teeth were incorrectly diagnosed as having caries $20 \%$ of the time. 


\subsubsection{Enhancement Techniques}

Current diagnostic methods initially segment dental X-rays into individual teeth in order to better judge if caries are present. A segmentation method is used to identify gaps between the teeth. Unfortunately this is sometimes problematic as X-rays can be noisy, have varying image quality or have artifacts present from the X-ray process. Research conducted by Ahmad et al. [12] tested four image enhancement techniques, namely adaptive histogram equalization (AHE), contrast adaptive histogram equalization (CLAHE), median adaptive histogram equalization (MAHE) and sharp contrast adaptive histogram equalization (SCLAHE). Their results concluded that using any of the processing techniques led to an improvement in image quality thus improving the correct diagnostic rates of caries in X-ray images with CLAHE and SCLAHE having the slight edge with respect to visual assessment whilst MAHE and AHE yielded better results on the contrast improvement index thus being better from a computer vision standpoint as described by Yoon et al. [26]. Bharathi et al. [27] considered the effectiveness of median, finite impulse response (FIR) and Gaussian filters in order to reduce noise levels in X-rays. Although their sample data represented extremes with respect to noisy data, they concluded a median filter obtained the best results.

\subsubsection{Segmentation Methods}

Both segmentation and feature extraction are vital stages required for caries detection. Unfortunately the majority of segmentation research focuses on tooth segmentation for the purposes of human identification and as a result, features required for caries detection are lost in favour of preserving crown shape in order to match teeth. Most segmentation methods converge to the use of integral projection in order to separate the individual teeth, however there are several approaches which can be implemented to optimize the integral projection results. Rad et al. [28] compiled an evaluation of various segmentation methods used when separating dental radiographs.

The first method discussed in their evaluation was segmentation using thresholding. This method was initially proposed by Nomir et al. [29] for use in dental imaging after adapting the works of $\mathrm{Hu}$ et al. [30]. The initial implementation of this method was for use in the automatic segmentation of CT lung images. This method was adapted 
for use with dental images as it accounted for the differences in contrast in Computerized Tomography (CT) images across multiple patients. A mask of the initial image is obtained by performing an iterative and adaptive threshold. Integral projection is performed on this mask based on the assumption that most, if not all, of the non teeth related pixels have been removed. It was concluded that this method was extremely fast and yielded positive results, however it was negatively impacted by noisy images and $\mathrm{X}$-ray artifacts, thus requiring pre-processing to be done. If the images allow for this method it proves highly effective, as in a later collaboration Nomir et al. [31] was able to use this segmentation method for human identification. Lin et al. [32] also used an adaptation of the method presented in Ref. [29] for use in human identification.

Several variants of this method have been proposed with most of the variation occurring in the acceptance criteria of the segmentation lines. Jain et al. [33] used integral projection for both vertical and horizontal separation of bitewing X-ray images thus resulting in the segmentation of X-rays into upper and lower jaw regions followed by the separation into individual teeth. A probability model defined by

$$
p_{v i}\left(D_{i} ; y_{i}\right)=p_{v i}\left(D_{i}\right) p_{v i}\left(y_{i}\right)
$$

Where $p_{v i}\left(D_{i}\right)$ is calculated by the integral projection of a given line subtracted from 1 all multiplied by a constant normalizing factor and $p_{v i}\left(y_{i}\right)$ is a Gaussian whose value is determined by the position of the previous segmentation line.

This was further developed by Frejlichowski and Wanat [34] with the primary focus of their research being on the development of a segmentation method for panoramic X-rays. Their method used a Bayesian probability formula, using the estimated width of the tooth as a guideline to determine the position of separation lines for tooth segmentation.

Segmentation through active contours was proposed by Zhou et al. [35] as a means to mitigate these problems and enhance results. Active contours are also used by Oliveira [16] after the segmentation process in order to obtain the boundary of individual teeth. Using the snake method developed by Kaas [36], an energy minimizing spline, which is guided by external forces and influenced by image forces, pulls towards an image's 
contours. The snake method regards the spline as if it were an elastic band thus the model aims to reach a minimum energy state. The formula to depict this is

$$
E_{\text {snake }}=\int\left(\alpha(s) E_{\text {continuous }}+\beta(s) E_{\text {curvature }}+\gamma(s) E_{\text {image }}\right) d s
$$

Where $\alpha, \beta$ and $\gamma$ are user defined constants that control the ability of the snake to change size, adhere to edges and its attraction to edges respectively. $E_{\text {continuous }}$ and $E_{\text {curvature }}$ represent the internal energy forces of the snake, forcing the snake to be a single contour and maintaining the degree of curvature it is permitted. $E_{\text {image }}$ represents the external energy force of the snake's attraction to the image edges.

This method handles segmentation and boundary detection simultaneously at no added processing cost [37] and is not adversely affected by noise. Despite these benefits, Ref. [28] concluded its tendency to adhere to strong image contrasts proved problematic for medical image segmentation. It is possible to perform active contours without edges as was demonstrated by Oliveira and Shah et al. [38], however when compared to other segmentation methods there was a distinct drop in the accuracy of the results.

Rad et al. [39] also proposed the use of k-means clustering as a means to achieve tooth segmentation. Teeth in the X-ray images are represented as clusters due to their paler intensity compared to surrounding tissue. The gaps between the clusters are equivalent to the gaps between teeth allowing integral projection to take place. Textural features along the cluster's contour were stored using a gray-level co-occurrence matrix. Unfortunately this method is affected harshly by noise and may create multiple clusters for a single tooth as a result.

\subsubsection{Caries Detection Methods}

The actual caries diagnosis relies heavily on assessing image properties along the boundary of each tooth. There have been several different approaches to how this is done which results in a divergence of analytical methods. Solanki et al. [40] used an unsupervised learning approach where they analyzed the shape contour of each tooth. This was achieved by converting X-ray images into binary images obtained from applying top-hat 
and bottom-hat filters to the initial X-ray and then applying a threshold. This process removed any dark regions along the boundary of the tooth which could have been caries areas. The shape of the tooth was then analyzed, flagging any areas where indentations along the contour of the tooth were present as caries markers. This method is effective in images with advanced caries where the distinction between tooth enamel and cavity is obvious, but fails to detect caries in the early stages of development.

Oprea et al. [41] proposed a binary threshold be applied on a high contrast image. A subsequent rule check was performed to determine if any black pixel groups occurred within the tooth or along its boundary and flagged these as caries.

Oliveira [16] made use of a supervised learning approach and developed a set of classifiers for caries detection. Statistical, region based, textural and boundary features were obtained by using an active contour method to isolate the boundary of each tooth after segmentation. This data was then obtained by a co-occurence matrix and compared against the classifiers which were obtained from a learning set where the presence of caries was known. Similarities between the test image and the classifiers resulted in a positive caries diagnosis.

Finally, Zhang et al. [42] used a blob detection method to isolate potential dental caries for 3-D rendering and assessment. This provided them with a good isolation of the caries from the affected tooth however it was sensitive to noise.

Typically k-means and c-means clustering algorithms are used when data is grouped in X-ray images [43], which would be the case when caries regions are defined. Fischer et al. [44] proposed that the use of a connectivity kernel resulted in a far lower error rate compared to these two methods. Their method involved introducing a transformation during the pre-processing stage in order to convert separate, elongated structures, into a single compact one. This was done by creating a bridge between the clusters in order to apply a connectivity kernel. Their method classified clusters correctly with an error rate of $1.5 \%$ compared to the $30.6 \%$ error rate that was present for k-means clustering method. 


\subsection{Conclusion}

Compared to the amount of research available with regards to image segmentation, relatively little has been explored with regards to caries diagnosis. Despite this, it is important to establish a successful segmentation method in order to maximize the potential for success later on. Several successful methods have been discussed, with the advantages and disadvantages noted. The key in selecting the correct method lies in the dataset which is analyzed.

Depending on which method is implemented, it is also important to note that an image enhancement technique may need to be implemented. In this section several cleanup methods were proposed with all of them obtaining similar outcomes. The choice of which one is used falls to processing time when compounded with the other methods which need to be implemented.

Finally, the process of isolating the caries regions was discussed. Once again, several methods were proposed, with the two major approaches being to either analyze the shape of the teeth to determine if caries are present, or to determine if there are any darker regions present which could indicate the presence of caries. Although both approaches are subject to interference from noise, they provide a means of obtaining the regions of interest.

The following chapters describe a caries diagnosis framework which implements and adapts the best methods described in this chapter. The proposed framework provides a new explores a new approach to caries diagnosis using image processing techniques and offers some insight into new techniques.

The next chapter details the first stages of the diagnostic method, namely the steps involved to separate the X-rays into individual teeth. 


\section{Chapter 3}

\section{Teeth Segmentation}

\subsection{Introduction}

The segmentation of dental X-rays into individual teeth is the most important step of any method which involves dental analysis algorithms [45]. If the teeth are correctly separated the subsequent stages are far more likely to yield correct results. The main difficulty is deciding on the correct method from those discussed previously. Important factors to take into consideration when deciding on a segmentation method include the noise levels of the images, as well as the presence of X-ray artifacts. In addition, the presence of fillings in the teeth means that there are abnormalities in the X-rays that would affect all of the proposed segmentation methods.

Taking these factors into account, the threshold method is implemented. This is due to the low noise levels of the dataset being analyzed, as well as the various artifacts and abnormalities that are present. This method is used as the pre-processing requirements are minimal whilst a strong segmentation method is implemented. In this chapter the adapted segmentation method is presented as well as the pre-processing steps involved to maximise its efficiency.

The pre-processing techniques used are covered in Section 3.2. An adaptation of the thresholding method described in [29] is discussed in 3.3 including the experimental results obtained while attempting to adopt this method. Finally the method implemented to obtain the best separation lines is discussed in Section 3.4. 


\section{$3.2 \quad$ Pre-Processing}

In order to optimize the diagnostic rate of the method discussed in this dissertation, it is crucial that the quality of the X-ray images being processed is optimized. In order to achieve this, image enhancement techniques are used to remove noise and improve the quality of the image. Before the image can be enhanced it first needs to be cleaned to remove any abnormalities which can arise from the radiograph process.

\subsubsection{Image Cleanup}

The iterative and adaptive thresholding techniques applied to the dental images require that only pixels relating to teeth, gums, jawbones and the background are present. In order to isolate these pixels, each image has a cleanup filter applied which removes any anomalies from the image. This is primarily related to the detection and removal of Position-Indicating device (PID) alignment artifacts which are defined later in the dissertation. The reference information generated at the bottom of each image by the dental X-ray software was also removed. The purpose of this reference information was to provide healthcare professionals with details relating to the radiograph they were viewing, however it served no purpose for computer vision techniques.

Figure 3.2 demonstrates the effect of the cleanup process on an image with both the reference information bar and PID alignment artifact present.

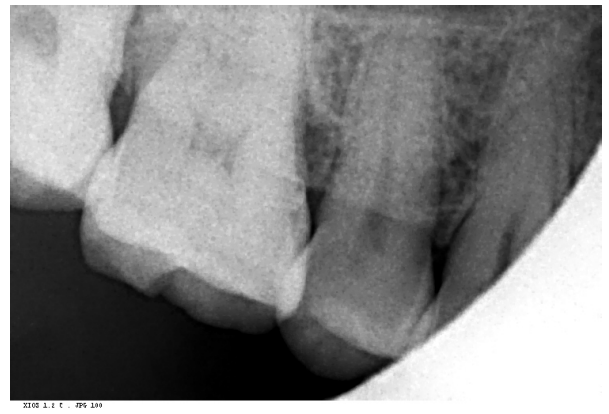

(a)

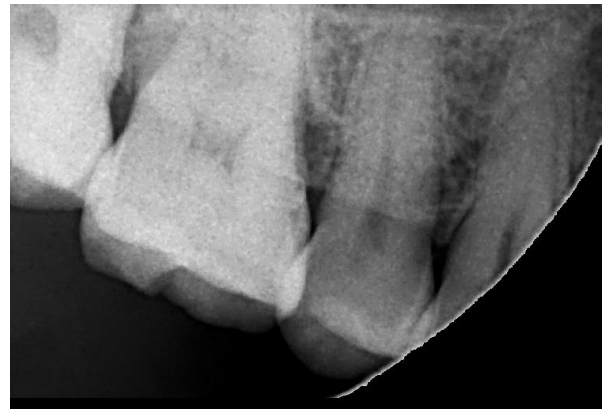

(b)

Figure 3.1: An example of the image cleanup process. (a) shows the original image.

(b) shows the image after artifact and reference information bar removal 


\subsubsection{Image Enhancement}

Once all non-organic structures barring dental fillings have been removed it is possible to balance the image contrast. In order to preserve feature detail, noise reduction obtained from blur filters was avoided. Utilizing the noise reduction techniques discussed in [26] and the enhancement techniques covered in [12], a median filter was initially applied to the image followed by a histogram equalization. The effects of this process can be seen in Figure 3.2. This also assisted in the impact of under- and overexposed images.

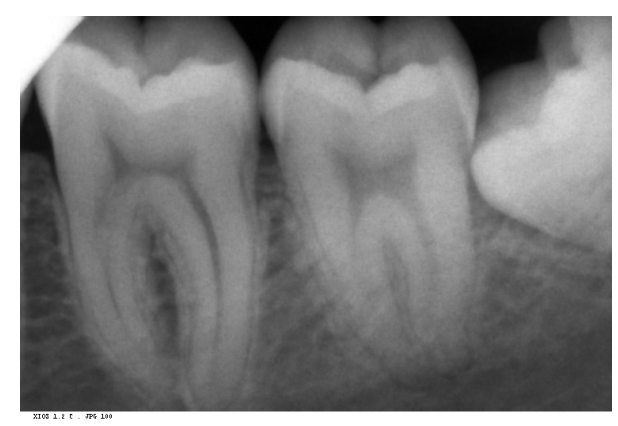

(a)

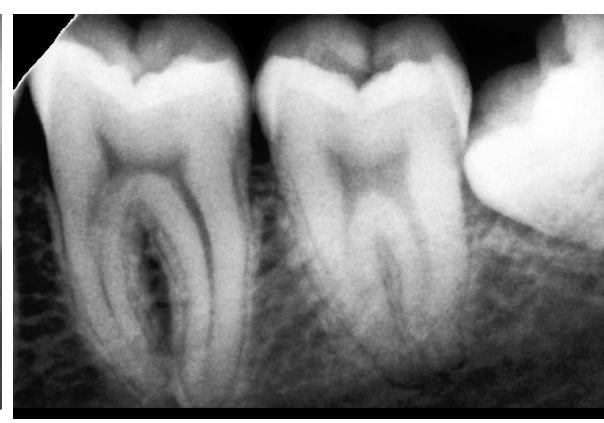

(b)

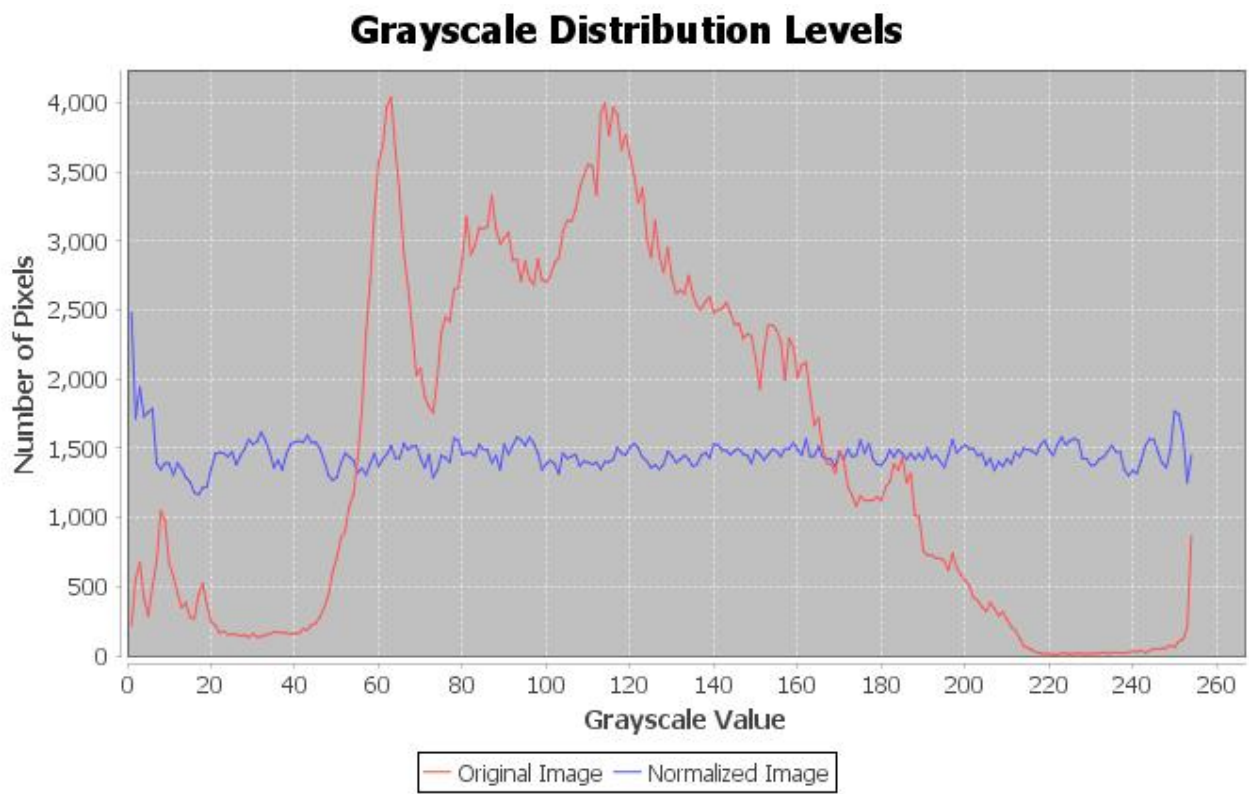

(c)

Figure 3.2: An example of the image enhancement process. (a) shows the original image. (b) shows the image after the median filter and histogram equalization have been applied. (c) shows the effects of the histogram equalization process 


\subsection{Thresholding}

\subsubsection{Iterative Thresholding}

Using the model proposed in [29] as the basis for the thresholding implementation, a canny edge detector was used to obtain the general outline of the teeth in the X-ray image. A morphological dilation was then applied to these edges in order to obtain the pixels in the area assumed to be the tooth boundary. Approximately half of the pixels obtained this way will correspond to the teeth and the other half will be of the jaw bone and other background objects. The initial threshold value was calculated from the average pixel value of the assumed teeth pixels and the background pixels and subsequent thresholds were calculated as follows:

$$
\begin{aligned}
\mu_{D}^{i} & =\frac{\sum_{(i, j) \in \text { dental }} f(i, j)}{\# \text { dental_pixels }}, \\
\mu_{B}^{i} & =\frac{\sum_{(i, j) \in \text { dental }} f(i, j)}{\# \text { background_pixels }}, \\
T_{i+1} & =\frac{\mu_{B}^{i}+\mu_{D}^{i}}{2} .
\end{aligned}
$$

Where $\mathrm{f}(\mathrm{i}, \mathrm{j})$ is the grayscale value of a pixel at point $(\mathrm{i}, \mathrm{j}), \mu_{D}^{i}$ and $\mu_{D}^{i}$ are the mean grayscale values for their respective regions and $T_{i}$ is the threshold value for the whole image calculated from the average values of the background and teeth pixels.

This step was repeated until the iterative threshold value did not change in subsequent re-evaluations or until a hard limit was reached. It was determined in Ref. [29] that convergence occurred within four to ten iterations for their set of images. Convergence occurred within seven to fifteen iterations for the images used in this dissertation, so a maximum iteration limit of fifteen was used. As it appears that the number of iterations is dependent on image size, a larger value could be used as a hard limit based on the size of the images being processed.

Once a final value has been obtained for the iterative thresholding stage a mask of the Xray was produced by isolating all teeth pixels whose grayscale value equaled or exceeded the iterative value. 


\subsubsection{Adaptive Thresholding}

Whilst the iterative thresholding process could be implemented without changes, the adaptive thresholding proposed in [29] did not work for the dataset used in this dissertation. Following standard adaptive threshold implementation, a pixel undergoes thresholding if, with it being the centre pixel of a window of size I x J pixels, its grayscale value is less than the mean value of all non-zero pixels within the window. The formula for this is thus

$$
T(i, j)=\frac{\sum_{s=-\frac{I}{2}}^{\frac{I}{2}} f(i+s, j+t)}{\# \text { nonzero_pixels }}
$$

Even with histogram equalization being performed prior to the thresholding phase stage, the remaining background pixels fell within the allowed variance of the teeth pixels. Furthermore, some of the teeth pixels appeared darker than the background pixels due to cavities and the removal of these regions would negatively impact the diagnostic algorithm later. A straight implementation of [29], whereby a training set of images was used to determine a global threshold for all masks resulted in too many of the background pixels remaining in the mask. Conversely, if only the pixels within the image were used to establish the threshold, much of the necessary mask was lost. Figure 3.3 demonstrates these two extremes.

In order to obtain a threshold value which correctly removed as much of the non-teeth pixels as possible a hybrid approach was used. A global threshold was determined by applying the adaptive threshold to the mask images of 40 images. The average of these thresholds was used to establish the global threshold value. The same process was applied to each image when it was undergoing thresholding to obtain its personal threshold. A final threshold value was obtained from the weighted sum of these two values where the distribution of the pixel intensity affected the weights. The initial weight for each threshold was set to 0.5 on the basis that the global threshold represented the average intensity trend for the dataset and that the personal threshold corrected any slight deviations. If there was a discrepancy between the two values, such that a $10 \%$ deviation or greater was present, the following rule was applied: 


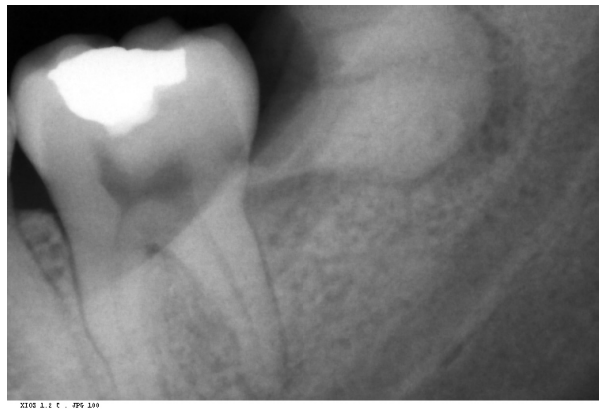

(a)

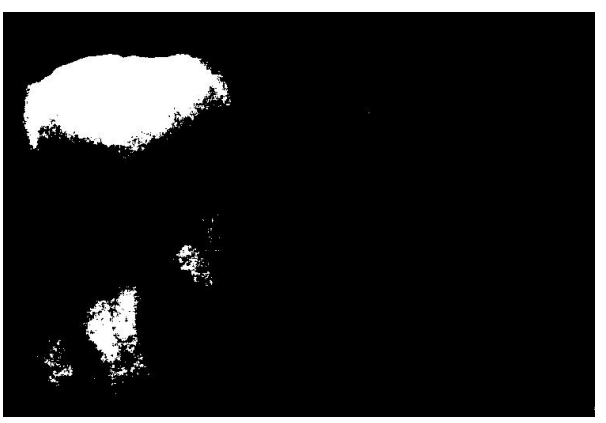

(c)

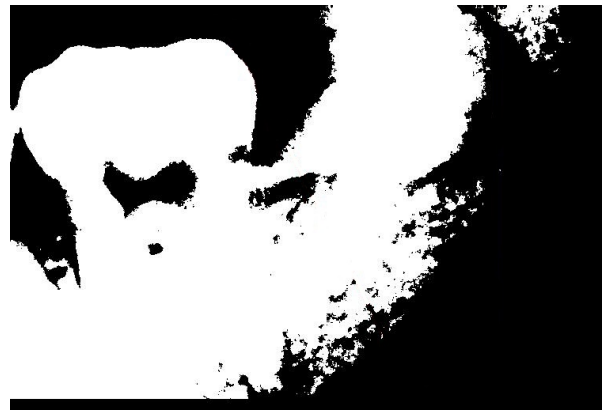

(b)

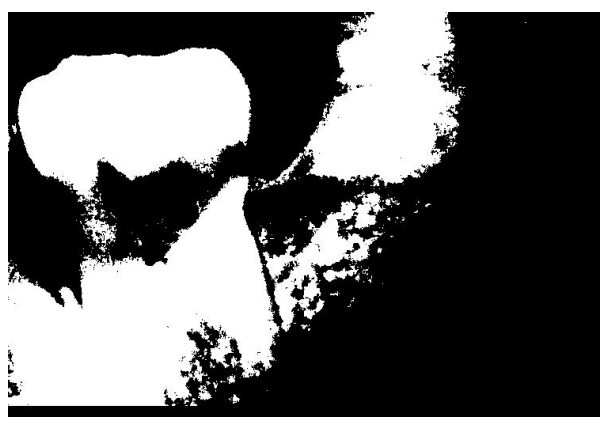

(d)

FiguRE 3.3: An example showing the results of the different approaches used to obtain a global mask threshold from adaptive thresholding. (a) shows the original image. (b) shows the result of generating a threshold from the global adaptive values. (c) shows the result of generating a threshold value from the image itself and $(d)$ shows the result of the hybrid approach

$$
F T(i, j)=\left\{\begin{array}{llc}
0.6 P A T+0.4 G A T, & \text { if } & 1-\frac{P A T}{G A T}>=0.1 \\
0.4 P A T+0.6 G A T & \text { if } & 1-\frac{G A T}{P A T}>=0.1
\end{array}\right.
$$

Where FT is the final threshold, PAT is the personal adaptive threshold and GAT is the global adaptive threshold.

\subsection{Tooth Separation}

\subsubsection{Integral Projection}

In order to separate the mask obtained from the previous stage, an integral projection method was implemented. The basis of integral projection is to analyze the pixel intensities across an image and detect regions of darker pixels. When the integral projection 
method was applied to the masks, the zero pixel regions that were removed during the previous stage were depicted as valleys as seen in Figure 3.4.
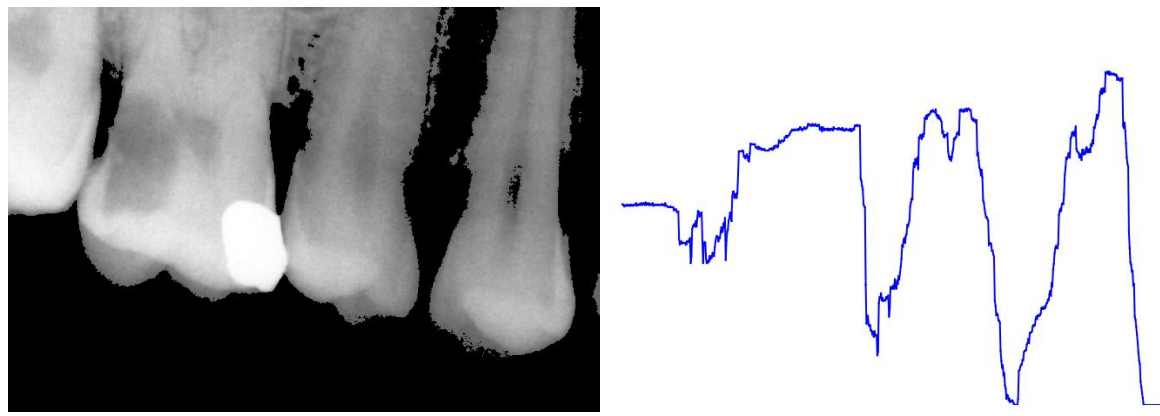

FigurE 3.4: An example of vertical integral projection detecting the gaps between the mask image of the X-ray

Due to the system not knowing if these masks pertained to upper or lower jaw images, all valleys were accepted at this point. This resulted in the acceptance of valleys depicting the crest between the root of some teeth as seen in Figure 3.5. This was handled in the next step of the algorithm.

To determine an initial separation line the centre of each valley was used as a spacing pixel to allow for the creation of dividing lines. These pixels were grouped into clusters denoting potential separation points within the image. For this specific dataset, clusters were determined as any group of blank pixels marked in the integral projection where there was not a vertical difference between subsequent points greater than $75 \%$ of the vertical height of the image nor a horizontal gap between subsequent points greater than $3.5 \%$ of the width of the image. Clusters were defined as any group of 40 or more pixels.

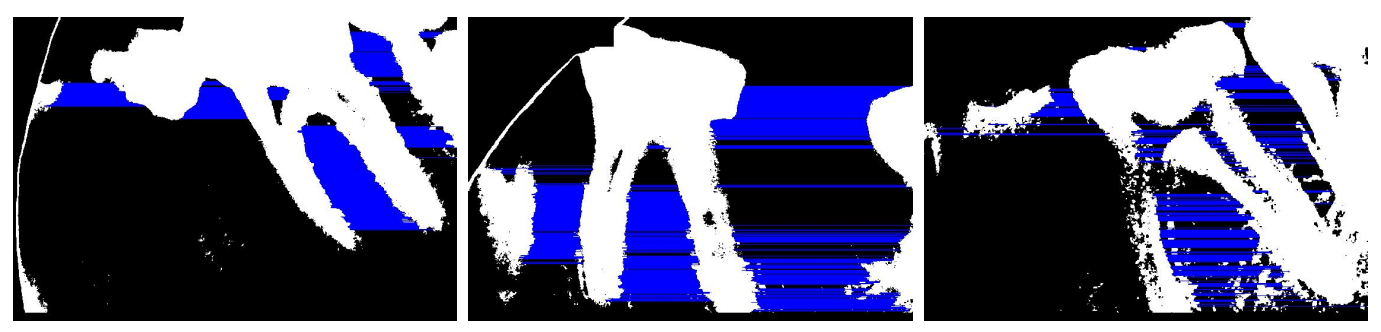

FIGURE 3.5: Examples of where valley detection incorrectly identified the crest between molar roots as a gap 


\subsubsection{Line Selection}

Separation lines were calculated using the gap clusters as training points for a linear regression model. Two variations of the simple linear regression algorithm were used. The first algorithm was the standard formula defined as follows:

$$
\begin{aligned}
\hat{\beta} & =\frac{\sum_{i=1}^{n}\left(x_{i}-\bar{x}\right)\left(y_{i}-\bar{y}\right)}{\sum_{i=1}^{n}\left(x_{i}-\bar{x}\right)^{2}}, \\
& =\frac{\sum_{i=1}^{n} x_{i} y_{i}-\frac{1}{n} \sum_{i=1}^{n} x_{i} \sum_{j=1}^{n} y_{j}}{\sum_{i=1}^{n} x_{i}^{2}-\frac{1}{n}\left(\sum_{i=1}^{n} x_{i}\right)^{2}}, \\
& =\frac{\overline{x y}-\bar{x} \bar{y}}{x^{2}-\bar{x}^{2}} \\
\hat{\alpha} & =\bar{y}-\hat{\beta} \bar{x} .
\end{aligned}
$$

Where $\mathrm{n}$ denotes the number of points, $\beta$ denotes the gradient of the slope and $\alpha$ denotes the y-intercept.

The second formula was a weighted linear regression model which proved effective in generating a correct separating line in cases where cluster distribution was favoured in one direction. If there was an equal distribution of points around the median then the simple linear regression model was used. If the distribution of points was greater or less than the median then the value of $n$ in the above equation was calculated to be half the total number of points.

In order to determine the best separation lines, the algorithm proposed in [34] was used as a starting point. The proposed algorithm works primarily due to the nature of panoramic X-rays, where all teeth are in view and as such it is able to use the uniform nature of teeth sizing to determine spacing. Due to the nature of the X-rays being analyzed in this dissertation, such an approach would not work if used verbatim. Despite this, by combining the rotation algorithms used in [29] and [33] with an altered probability model of [34], it is possible to develop a combination of a rotational and probabilistic algorithm for segmenting the dataset outlined above.

Initial lines obtained from this step separate each tooth by a rough estimation of the gap angle. In order to determine the best angle of rotation, a non-greedy selection 
algorithm was implemented. Using the centre point of the gap as a rotation point, the separation line underwent a rotation between -20 to 20 degrees in increments of 0.25 degrees generating 160 iterations of potential segmentation line candidates. The original line obtained by a rotation of 0 degrees acts as a benchmark to judge the improvement of each rotational step. A multi-check acceptance algorithm was used to determine the line of best fit using the number of teeth pixels intersected and their ratio to the total line length as a basis for acceptance. Let $P$ refer to the number of teeth pixels hit by a separation line, where the best refers to the minimum number of points hit. The horizontal distance covered by the line in pixels is represented by $D$. $w$ is a width constant, in this case defined as 0.3 and $c$ is a constant defined as 0.9. Let the total length of the line be represented by $L$ and finally, let the percentage of teeth pixels intersected by the line relative to the line itself be represented by $H$. Using these symbols, the process for line selection is outlined in Algorithm 1.

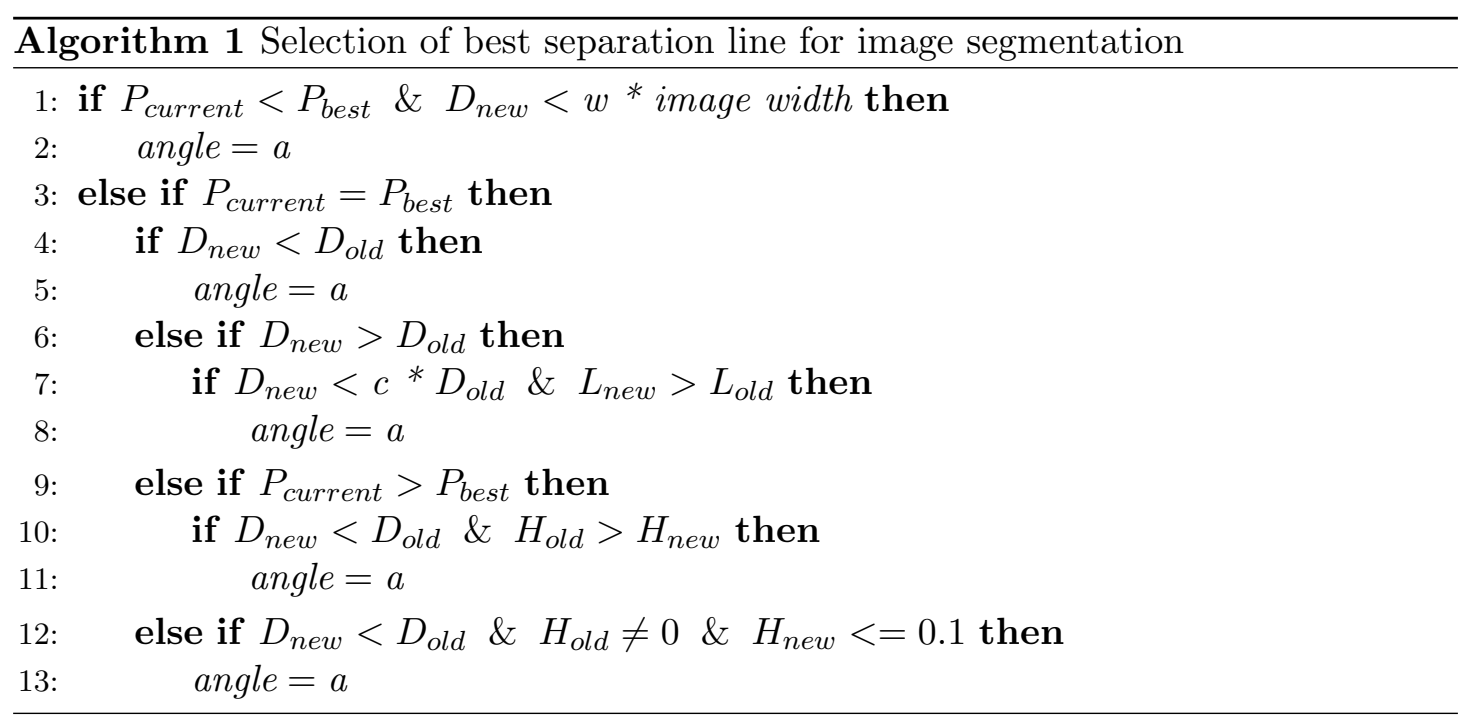

The primary check done by this algorithm is to determine if segmentation lines with fewer intersection points are not bypassing the gap between teeth as seen in Figure 3.6. For segmentation lines where the number of intersection points is equal to, or greater than, a previously determined optimal line, a new set of acceptance criteria were introduced. Based on the probability formula implemented in [33], vertical lines have a higher probability of generating successful segmentation results. The weighting system judged potential line candidates by using slope gradient and intersection point percentage relative to the total separation line. The probability of a line being the best segmentation line was determined by 


$$
P=\left|n W_{T}+\frac{W_{I}}{I P_{\text {deep }}}-J\right|
$$

Where $\mathrm{P}$ is the probability of the line being correct, $\mathrm{n}$ is the number of already segmented teeth, WT is the assumed width of the previous teeth, WI is the width of the image, IP are the number of integral projection points representing gaps between the teeth and $J$ is the projected point of the segmentation line. The desire of the algorithm was to minimize the value of $\mathrm{P}$ where $\mathrm{P}$ actually represents the probability of a line being incorrect. Lines which fall on the projected segmentation value have a $\mathrm{P}$ rating of 0 meaning there is close to 0 probability of it being incorrect. As segmentation lines move away from the projected point the value of $\mathrm{P}$ increases resulting in unfavourable selection chances.

In order to accept separation lines for impacted or extremely close adjacent teeth, a second algorithm was used. If more than $60 \%$ of the separation line intersected with teeth pixels then the line was discarded and the gap was regarded as a space between molar roots. Figure 3.7 shows how this rectifies the issue caused by the acceptance of these gaps shown in Figure 3.5.

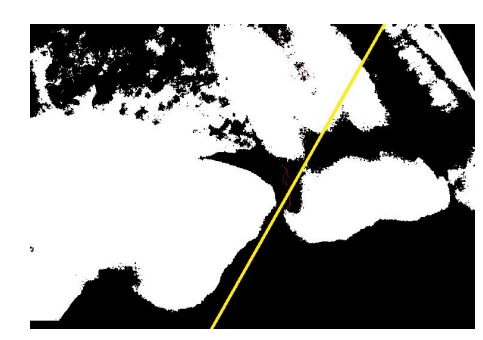

FiguRE 3.6: An example of where the separation line attempts to bybass the gap between teeth in favour of a line with fewer intersection points
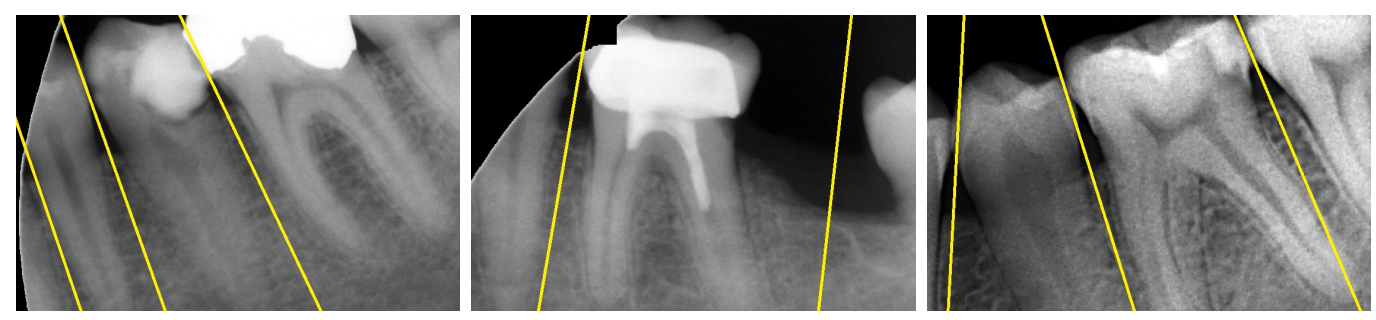

Figure 3.7: Examples of how the crest detection algorithm correctly removed the separation lines for the images in 3.5 


\subsection{Conclusion}

In this chapter the implementation of a segmentation method has been discussed. Due to the thresholding approach being selected the pre-processing methods implemented to ensure accurate segmentation were also discussed. In order to deal with the nature of the X-rays being processed a hybrid method for generating the initial threshold values was introduced. Furthermore, a new acceptance algorithm has been proposed for separation line acceptance which uses the valleys of the integral projection to generate a probability acceptance formula.

In the next chapter a boundary detection method using the results of this section is presented. The various steps involved with isolating the search region for caries identification will be discussed in more detail. 


\section{Chapter 4}

\section{Tooth Boundary Detection}

\subsection{Introduction}

In order to properly assess the presence of caries, the boundary of each tooth needs to be analyzed. Results obtained from the previous stage of the proposed diagnostic model consist of segmented images of individual teeth. As was shown in the previous chapter, although the X-rays were correctly segmented and attempts were made to reduce the amount of background pixels present, there are still non-tooth pixels present in these images. At this stage the tooth pixels which are present relate only to a single tooth so no further cleanup is required to isolate each tooth. The presence of these nontooth pixels affects the boundary detection algorithms as the exact contour of the tooth is unknown. Furthermore, once the boundary has been determined, the search space needs to be minimized so as not to process points closer to the centre of the tooth where the contrast is darker.

In this chapter the implementation of a pre-processing technique to remove the non-tooth pixels is covered in Section 4.2. The implementation of an active contour method for boundary detection is proposed in Section 4.3 along with examples of its effectiveness. Finally, Section 4.4 discusses the implementation of two different algorithms used to minimize the search space and their relative effectivness. 


\subsection{Top and Bottom Hat Transformations}

As evidenced from the previous results, a cleanup of the segmented images is needed in order to remove any non-tooth pixels which may affect boundary detection. A top and bottom hat transformation was proposed in [16] as a possible solution to this problem. Due to the similarity of the images obtained from the previous stage and those in [16], this method was adopted. In order to increase the contrast of the image, thus isolating the brighter areas of the image where dental matter is located, a composite transformation was used as follows:

$$
I_{\text {output }}=\left(I_{\text {Original }}+T H\right)-B H
$$

Where $\mathrm{I}_{\text {output }}$ is the desired output image, $\mathrm{I}_{\text {Original }}$ is the original image obtained from the previous segmentation process, $\mathrm{TH}$ is the top hat transformation applied to the original image and $\mathrm{BH}$ is the bottom hat transformation applied to the original image.

The output was obtained through the addition and subtraction of pixel values obtained from these transformations. Any negative value was set to 0 and any number exceeding 255 was set to 255 in order to maintain the grayscale nature of the X-ray. In Oliveira's original proposal, the above algorithm was applied again on the initial output image. When this same approach was applied to the dataset used in this dissertation the degree of image loss proved too substantial to continue image analysis. This image loss was due to the contrast difference of the two datasets, where paler images require the second pass in order to ensure correct cleanup. Additional testing revealed that any images of poor quality, regardless of contrast, suffered definition loss when a two pass method is used. The use of a single pass method more closely resembles that proposed by [35], which was used during their segmentation stage.

Figure 4.1 demonstrates the application of this transformation where the removal of background pixels can be clearly seen. In addition to the removal of background pixels, the implementation of this transformation accentuates any caries by increasing the image's contrast. 

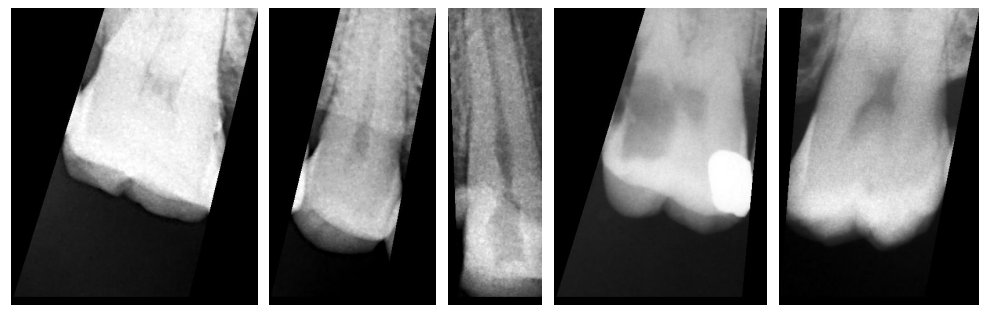

(a)
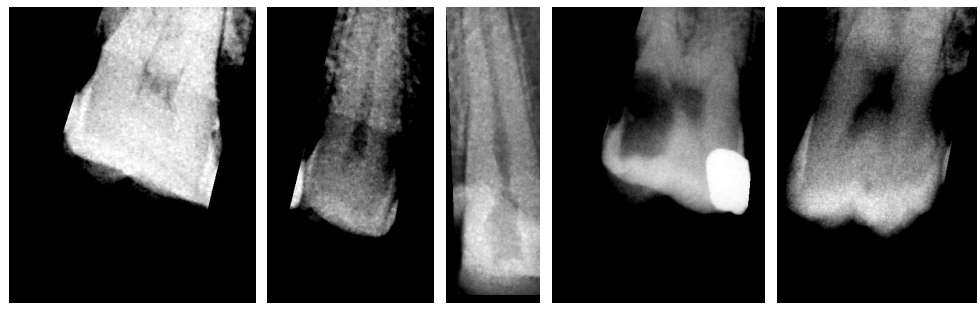

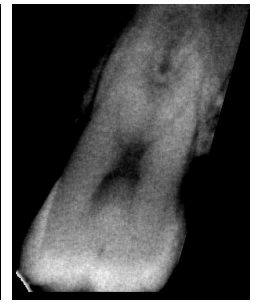

(b)

Figure 4.1: Examples of the top and bottom hat image transformations. (a) shows the images after their separation in the previous chapter. (b) shows the images after the transformation

\subsection{Boundary Detection}

Before caries detection can occur, the boundary of the tooth needs to be defined. By this stage most of the non-tooth pixels had been cleared but the inability of performing a second pass of the image transformation meant that residual background elements existed in images where the tooth had a low contrast difference. In order to remove these remaining elements and prepare the image for boundary detection with an active contour implementation, a binary filter was applied to each image. The threshold value for the binary filter was determined to yield best results when set to a the low percentile intensity value, thus removing $80-90 \%$ of all remaining background elements. Experimentation indicated that the best results were obtained when the threshold was set to ignore all pixels falling under the 15 th percentile.

As a result of implementing a binary filter in this manner, a simple edge detector could not be used. Due to the effects of the top and bottom hat transformation, narrow teeth such as those depicted in Figure 4.2 suffered degradation of their connecting pixels due to the darker contrast of the dental pulp located in their centre. The implementation of active contours proved to negate this problem. Following the formula outlined in Equation (2.2), a snake contour model was implemented on the binary image. The internal energy component of the snake model is responsible for ensuring a continuous 
curve is obtained. An expansion of the internal energy formula shows the elasticity and curvature components. By isolating the elasticity portion of the formula and isolating the $\alpha$ value, it can be seen that by setting an appropriate $\alpha$ value it is possible to limit the elasticity of the curve. The following formula demonstrates how this component is derived and how a sufficiently high $\alpha$ value negates the impact of the distance between the points on the curve thus limiting the elasticity of the curve.

$$
\begin{aligned}
E_{\text {internal }} & =\sum_{i=0}^{n-1} \alpha\left\|v_{i+1}-v_{i}\right\|^{2}+\beta\left\|v_{i+1}-2 v_{i}+v_{i-1}\right\|^{2}, \\
E_{\text {elastic }} & =\alpha \sum_{i=0}^{n-1}\left(\left(x_{i+1}-x_{i}\right)^{2}+\left(y_{i+1}-y_{i}\right)^{2}-\bar{d}\right)^{2}, \\
\text { where } \quad v_{i} & =\left(x_{i}, y_{i}\right)
\end{aligned}
$$

Where $d$ is the average distance between pairs of points on the curve.

A high value for $\alpha$ was used to disregard the space between the binary thresholded segments, with a value of 3 being used for this dataset. To compensate for the snake's reduced ability to shrink as a result of this elasticity restriction, the initial search space was set to an elliptical region 1.5 times the height and width of the binary sections. This approach negatively impacts the curvature of points around the roots of molar teeth as seen in Figure 4.3(a) however the search space refinement method described in the next section negates this problem. Figure 4.3(b) demonstrates the effectiveness of the method at isolating boundaries on non-molar teeth.
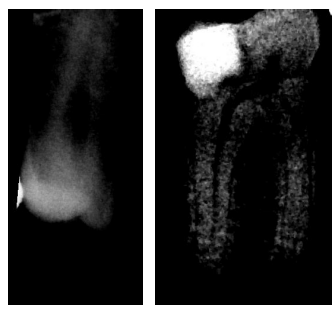

(a)

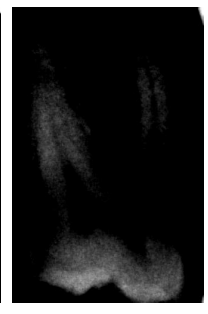

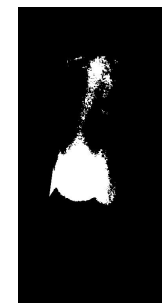
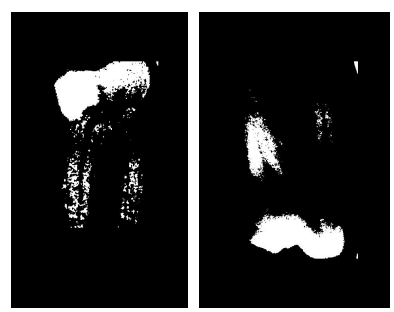

(b)

Figure 4.2: Examples of image degradation due to image transformations and binary thresholding. (a) shows the images after their top and bottom hat transformations. (b) shows the images after the binary threshold 


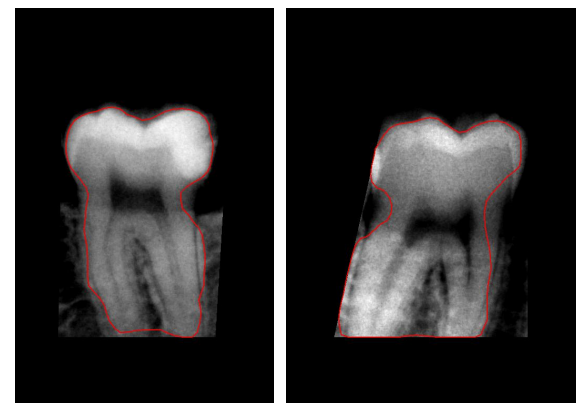

(a)
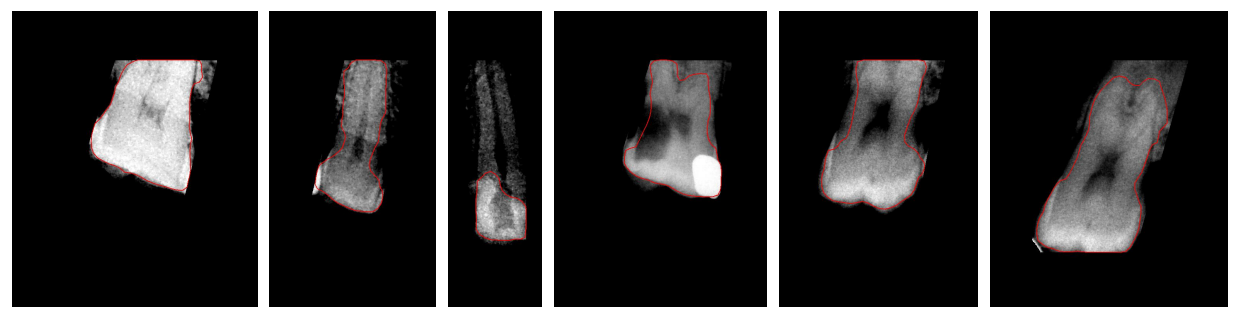

(b)

FIGURE 4.3: Examples of boundary detection using active contours. (a) shows images of molar teeth where the curvature of the boundary around the root is not perfect. (b) shows images of boundaries detected on other teeth

\subsection{Search Space Refinement}

\subsubsection{Centre Elimination}

When the images from the previous step were initially analyzed, a high false positive rate was encountered. This occurred when the caries detection algorithm started to analyze regions of the tooth close to the dental pulp where the contrast of the image is darker. In order to avoid these false positives, only pixels along the outer region of the tooth, nearest the boundary obtained from the active contour method, could be analyzed.

A method was investigated which focused on eliminating the centre of the tooth as a search region in order to avoid these false positives. To eliminate the correct regions, the orientation of the tooth needed to be determined. This was done so that potential caries along the crown of the tooth could be assessed and points along the neck and root region could be disregarded. The orientation was determined by analyzing the shape of the boundary region defined previously. A closing function on the boundary of the tooth was performed to calculate solid distance, the widest side of the boundary, where the width was determined by the number of adjacent pixels ignoring zero pixels, was assumed to 
be the crown of the tooth. For teeth where a gap was present when this distance was calculated, this region was assumed to be the root section of molar teeth. Using this information, a centre area was defined for exclusion in the detection algorithm. Testing determined that this area yielded best results when defined as being $20 \%$ of the width and $60 \%$ of the height of the tooth. Initially this proved effective in images where the orientation of the tooth was near vertical, however in angled teeth, or teeth that were partially cut off by the end of the slide, the centre was often misaligned as seen in Figure 4.4. In images where this occurred the problem of false positives was not diminished as seen in 4.4(b) where the correct search space was not identified.

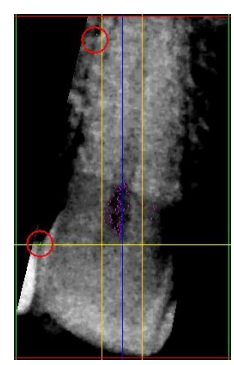

(a)

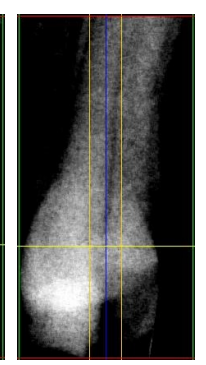

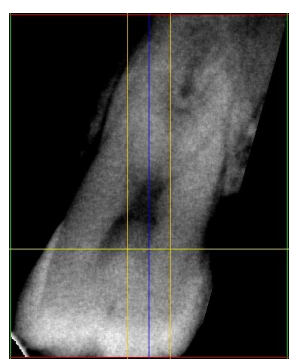

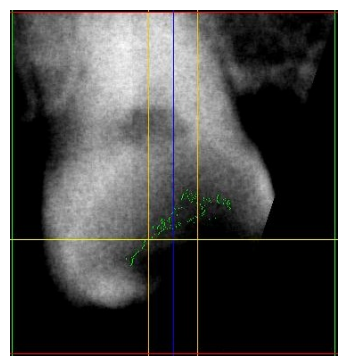

(b)

FiguRE 4.4: Examples of the centre elimination method. (a) shows examples of where this method correctly identifies the centre region of each tooth. (b) shows examples of where the centre region is defined incorrectly or results in known caries to be excluded

\subsubsection{Boundary Dilation}

Instead of using the centre as a reference point for boundary refinement, a second method was developed which aimed to rectify the problems of the first. This proposed method defines the search region by using the edge of the tooth as a reference point and thus is unaffected by tooth alignment. A dilation filter was applied to the boundary of the tooth, such that only dilation towards the centre of the tooth was obtained. The search region was confined to assess pixels adjacent to the outer boundary. In order to avoid the dental pulp the search region needed to be defined to only extend a certain depth. Experimentation showed that pixels varying between a depth of $10-15 \%$ from the edge of the boundary did not suffer from the same false positive rates as when the whole tooth was analyzed. This depth was dependent on the tooth, with smaller teeth requiring the depth of $10 \%$ and larger teeth obtaining favourable results with an analytical depth of 
$15 \%$. In order to determine this percentage, the height and width of the tooth were used as a scaling metric defined by

$$
\text { Dilation }=8+7(w / h)
$$

Where $w$ is the width of the tooth and $h$ is the height of the tooth. If the dilation value exceeded $15 \%$ in horizontal teeth, which occurred when impacted teeth were analyzed, the value was capped to $15 \%$. Figure 4.5 shows how this method achieves a better success rate at avoiding points along the inner region of a tooth whilst not missing points along the edge. In order to determine tooth orientation, the method proposed in the previous centre elimination method was implemented.
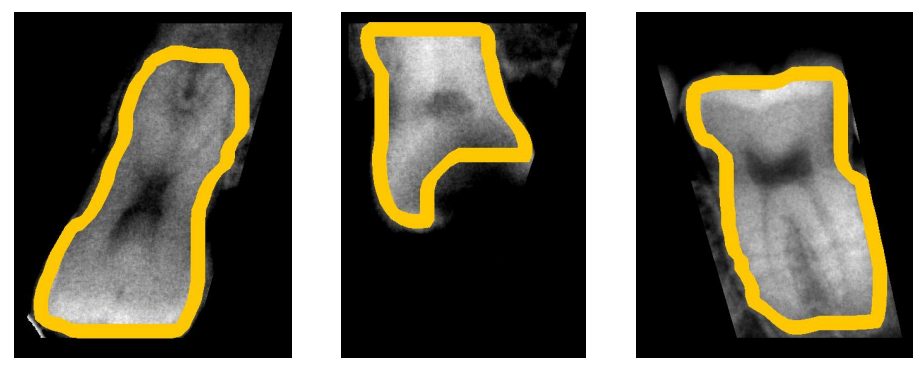

FIGURE 4.5: Examples of the boundary dilation method for search space refinement.

\subsection{Conclusion}

In this chapter, the implementation of a boundary detection method and search refinement method were discussed. The proposed adaptations to the top and bottom hat transformations in this chapter allowed for the removal of background structures, while providing a contrast enhancement to aid the caries detection algorithm which is proposed in the next chapter. The method used for boundary detection utilized an active contour model to determine the edges of the segmented teeth. This model was constrained by limiting the elasticity component of the algorithm in order to compensate for incomplete threshold values. Finally, two methods were proposed in order to refine the search space for the next process. Although the centre elimination method did yield some positive results as was demonstrated, ultimately the boundary dilation method achieved a higher success rate. 
The next chapter will present a caries detection method to be used on the search space defined in this chapter. This will conclude the diagnostic model proposed in this dissertation. 


\section{Chapter 5}

\section{Dental Caries Detection}

\subsection{Introduction}

In order to diagnose the presence of caries, the potential regions of interest must be defined. Once these regions are established, the diagnostic method can evaluate whether the region is of concern. This chapter introduces both the edge detector used to locate potential caries, as well as the novel method for diagnosing if caries are present in those regions. The algorithms for edge detection, caries region of interest highlighting and caries assessment are discussed.

Section 5.2 presents the blob detection algorithm used to obtain an initial position of suspected caries regions. Section 5.3.1 explains how the search space is extended to allow for image analysis techniques to provide an alternative to supervised learning models, whilst Section 6.5 covers the proposed diagnostic method and its implementation.

\subsection{Blob Detection}

The search space defined by the previous method represents the region where detectable caries can be located. The implementation of a blob detector allows for the detection of regions where dental caries may be present. Due to the high contrast of the image at this stage of the process due to the top hat transformation performed earlier, caries appear as substantially darker regions compared to the tooth matter. This favours blob detection algorithms, owing to their ability to locate local maxima. In order to obtain a 
region of interest which was not affected by scale due to the varying sizes of the teeth, the blob detection model proposed by Lindeberg [46] was implemented. This model uses a Laplacian of the Gaussian approach to detect darker regions, which is defined as a convolution kernel of the form

$$
L o G=\frac{x^{2}+y^{2}-2 \sigma^{2}}{\sigma^{4}} e^{-\frac{x^{2}=y^{2}}{2 \sigma^{2}}}
$$

Where $\sigma$ is the width of the kernel. This was approximated to a $5 \times 5$ kernel for the purposes of implementation defined as

$$
L o G=\left[\begin{array}{ccccc}
0 & 0 & 1 & 0 & 0 \\
0 & 1 & 2 & 1 & 0 \\
1 & 2 & -16 & 2 & 1 \\
0 & 1 & 2 & 1 & 0 \\
0 & 0 & 1 & 0 & 0
\end{array}\right]
$$

Any regions of sufficiently darker contrast were highlighted by the blob detector as seen in Figure 5.1. The darker pixels in these regions were then isolated into clusters using a connectivity kernel described in Figure 5.2. The use of a 4 way kernel resulted in some loss of definition around the edges of the caries clusters which negatively impacted the diagnostic method, therefore the 8 way kernel was implemented. Figure 6.7(b) shows the clusters obtained using the connectivity kernel.
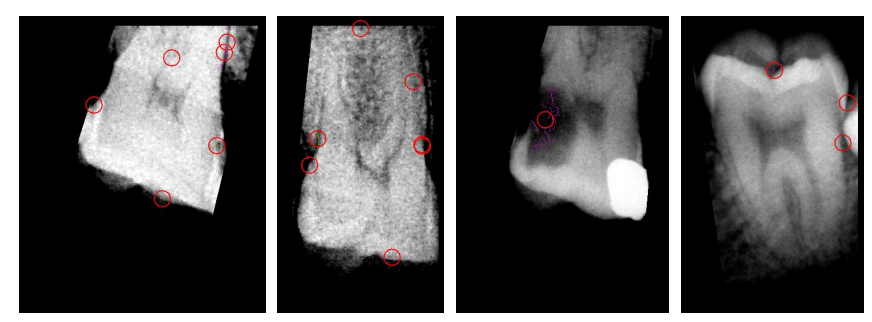

Figure 5.1: Examples of images displaying potential caries sites highlighted by the blob detector 

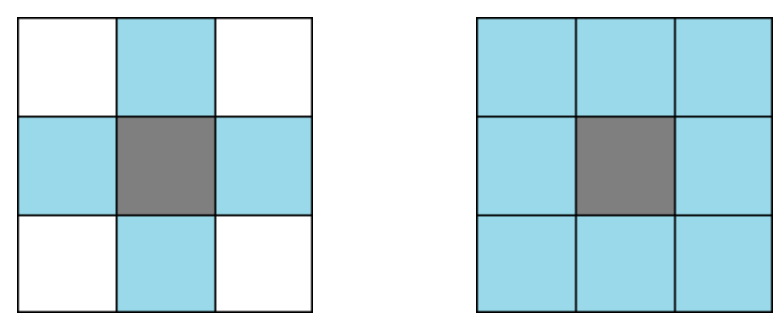

FiguRE 5.2: The two types of connectivity kernels which can be used for connectivity clustering
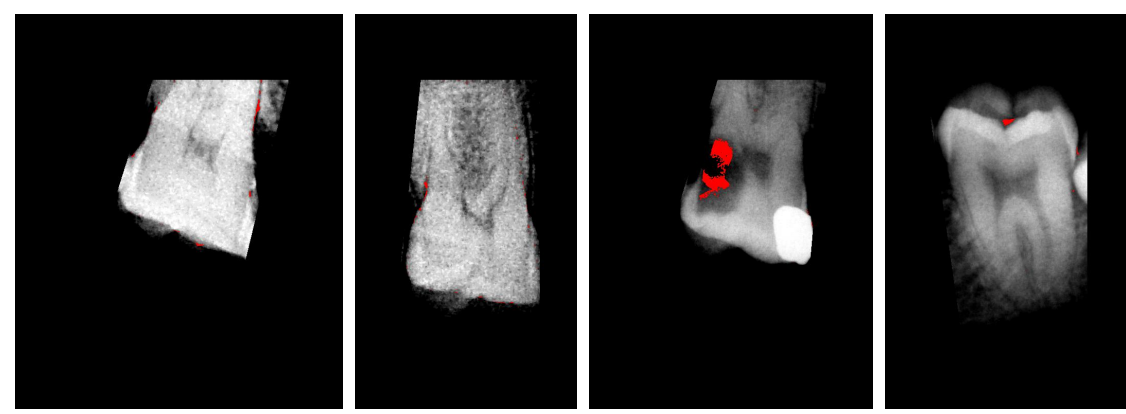

(a)
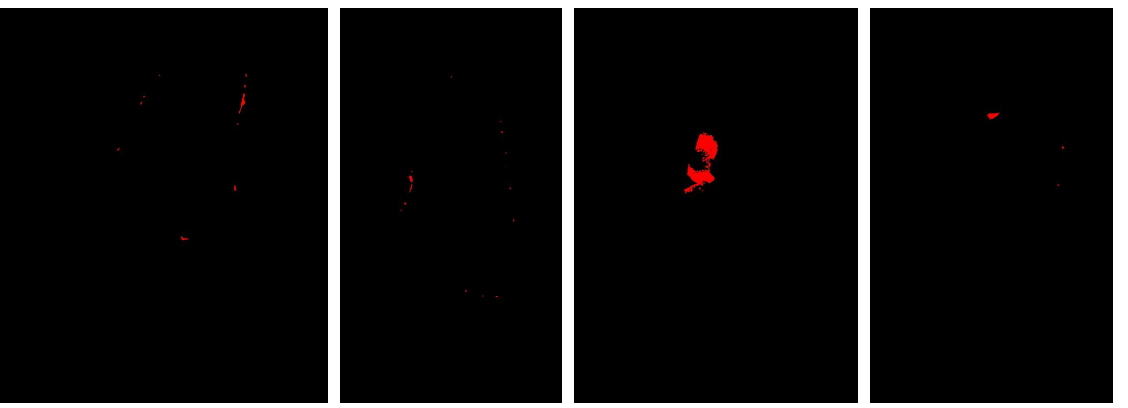

(b)

FiguRE 5.3: Examples of the caries clusters obtained from the connectivity cluster kernel (a) shows the clusters on the X-rays. (b) shows the isolated clusters

\subsection{Caries Analysis}

\subsubsection{Region of Interest Generation}

In order to diagnose whether dental caries were present using a non-supervised approach, standard dentistry analysis techniques needed to be implemented. The depth of the search region was already known relative to the tooth, falling between 10-15\% of the overall width. Average tooth sizes defined in [47] approximate teeth to range between 
7.5-9.0mm in width. As the images being processed are not panoramic X-rays, the exact tooth being analyzed was unknown as there is no way to predict distribution. In order to approximate the width, the following formula was proposed:

$$
W=T \max -\frac{T_{\text {variance }}\left(P_{\text {max }}-P_{\text {calculated }}\right)}{P_{\text {variance }}}
$$

Where $W$ is the estimated width, $T$ is the width of the tooth and $P$ is the percentage depth of the search space, determined to be $10-15 \%$ in the previous chapter. $T_{\text {variance }}$ is obtained by calculating the difference of the maximum and minimum tooth width values and is determined to be 1.5. The value for $P_{\text {variance }}$ was calculated to be 5 following the same process. This formula was derived based on the probability that teeth which require smaller analysis regions represent the narrower spectrum of teeth, whereas teeth with wider search regions represent the wider spectrum.

In order to obtain optimal diagnostic results, the current images, which still depicted the top and bottom hat transformations, were unsuitable due to the pixel loss. To rectify this, a normalized image of the tooth being processed was generated using the original X-ray. This enhanced the contrast to assist the diagnostic algorithm but did not reduce the amount of detail in the image. This was done due to caries detection being optimized in high contrast images where the contrast is neither too light, or too dark as outlined in [48]. The potential caries clusters on the high contrast images were used to denote the location of potential caries on the normalized image.

\subsubsection{Cluster Analysis}

To determine whether a cluster of pixels fit the criteria for caries classification, several acceptance factors were calculated. Firstly, a threshold value was obtained by calculating the mean pixel value of the cluster region. Next, a second threshold value was obtained by calculating the mean pixel intensity of the area surrounding the suspected caries region. Due to caries originating in the enamel of the tooth the search space was best constrained to this region as the dentin appeared darker in X-rays. Enamel thickness varies from $0.87-1.45 \mathrm{~mm}[49]$, thus the search space was obtained by creating an elliptical 
region centred along the perpendicular of the cluster with width equal to double its height and height defined by:

$$
H=E_{\text {max }}-\frac{E_{\text {variance }}\left(T_{\text {max }}-T_{\text {calculated }}\right)}{T_{\text {variance }}}
$$

Where $\mathrm{H}$ is the height of the ellipsis, $\mathrm{E}$ is the width of the enamel and $\mathrm{T}$ is the width of the tooth. $E_{\text {variance }}$ is obtained by calculating the difference of the maximum and minimum enamel width values and was determined to be 0.58 .

This confined the mean threshold generation to only neighbouring pixels within the enamel region. At this stage the two values were compared to determine whether there was significant variance between the two regions to facilitate further analysis. If the cluster was less than $5 \%$ darker than the surrounding area the cluster was discarded and no caries were identified. If the cluster region had a mean more than $15 \%$ darker than the surrounding area the cluster was identified as a caries region. If the cluster mean was between $5-15 \%$ darker, the algorithm proceeded to determine if the cluster represented a darkening of the X-ray itself or a section of early caries development. A Sobel operator with a kernel size of 3 was applied to the elliptical region in order to detect significant gradient changes within the region. By limiting the kernel size to the minimum possible, it was possible to apply the operator to any sized region of interest. In order to deal with the inaccuracies inherent to $3 \times 3$ Sobel kernels, the Scharr function [50] was used, defined as two kernels of the form

$$
G_{x}=\left[\begin{array}{ccc}
-3 & 0 & +3 \\
-10 & 0 & +10 \\
-3 & 0 & +3
\end{array}\right] \quad G_{y}=\left[\begin{array}{ccc}
-3 & 10 & -3 \\
0 & 0 & 0 \\
+3 & +10 & +3
\end{array}\right]
$$

These kernels were applied to all pixels within the analysis region, resulting in the transformation of

$$
G=\left|G_{x}\right|+\left|G_{y}\right|
$$


Where $\mathrm{G}$ is the value of the new pixel. If no edges are detected using this algorithm, it implies that there are no regions of significant pixel intensity change within the search region. As such, the cluster is regarded as a darkening of the X-ray which was initially flagged due to the exacerbated contrast brought on by the top and bottom hat transformations. If, however, an edge is detected this represents a region of pixel intensity change not in line with the surrounding area. As such these areas are denoted as caries.

\subsection{Conclusion}

In this chapter the proposed caries diagnostic algorithm was discussed. The main complication of the method is the definition of the search space, which was isolated by the combined use of a blob detector and connectivity clustering model, as well as the preceeding methods outlined in this dissertation. The final evaluation region of the area surrounding the caries cluster is determined using the proposed methods for determining tooth width and enamel thickness. Finally a sobel filter, implementing a Scharr function, was utilized to determine the presence of caries.

The next chapter will discuss the results of both the segmentation method and the diagnostic method and compare their results against current systems. 


\section{Chapter 6}

\section{Results and Discussion}

\subsection{Introduction}

In order to evaluate the performance of the overall method proposed in this dissertation, the individual success rates of the segmentation process and the caries diagnosis process need to be analyzed. Furthermore, it is important to understand the nature of the dataset which is analyzed to achieve these results.

Results pertaining to the different dental segmentation methods are readily available, therefore a comparison of the proposed method and its various algorithms, both adapted and novel, against existing segmentation methods were easy to obtain. In order to determine the performance of the caries diagnostic algorithm, comparisons needed to be made against the correct detection rate of both existing systems and dentists' ability to diagnose caries. Unlike the segmentation method evaluation, the ability to determine the failure rate of the diagnostic method relies on the ability of existing methods to detect caries. As such, the algorithm's performance was judged by its ability to locate known caries and any predictions it made with regards to caries which had not been previously identified were considered as false positives.

In this chapter information pertaining to the dataset [51] is presented in Section 6.2. This will cover any properties which may negatively impact the caries prediction rate and explain why they are present. Section 6.3 will outline the proposed detection framework described in the previous chapters. Section 6.4 will evaluate the segmentation results 
and discuss how they compare to existing research. Finally, Section 6.5 will discuss the results and comparative effectiveness of the caries identification method.

\subsection{Dataset}

\subsubsection{X-Ray Errors and Artifacts}

There are many different errors and anomalies which can affect dental X-rays. Radiographic errors refers to errors in the final X-ray due to incorrect human input whilst artifacts refers to any structures in a radiograph which should not be present under normal circumstances. Radiographic errors can occur either due to technical errors which occur during the capture process, or they can be errors that occur during the processing phase. Similarly, artifacts can occur as a result of errors during the processing phase and can be a result of improper handling of the film packet used to obtain the X-ray. Below are the details of the errors and artifacts relevant to the dataset.

\subsubsection{Missing Crown}

This error can also refer to partial teeth being present. This occurs when the film is incorrectly placed during the X-ray process resulting in the area of interest not being shown as seen in Figure 6.1. This is problematic during the diagnostic process as incorrect boundary depths are assumed and potential caries are missed as they are not in the X-ray.

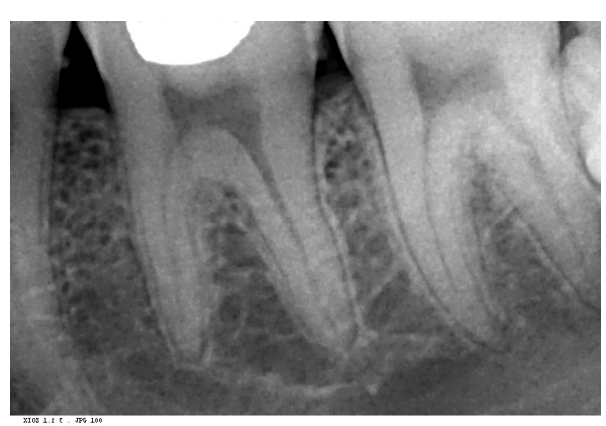

Figure 6.1: Example of dental X-ray with missing crown 


\subsubsection{PID Alignment Artifact}

Position-indicating device alignment artifacts occur when the PID is improperly aligned, thus causing the film to be incompletely exposed to the radiation beam. This results in a partial image on the X-ray, referred to as a cone-cut. An example of this can be seen in Figure 6.2

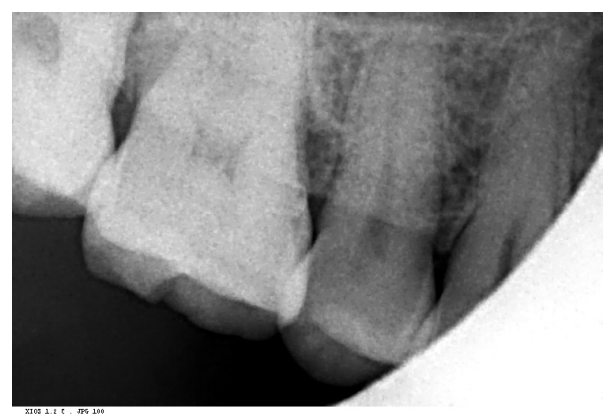

FIGURE 6.2: Example of a PID alignment artifact

\subsubsection{Exposure Errors}

There are two possible exposure errors, both of which affect the ability to analyze Xray images. Underexposed film is pale and the teeth in the image appear translucent. If this occurs there may be an issue with the milliamp seconds (mAs), which affects the darkness of the X-ray, the kilovolt peak, which affects the contrast of the image, the exposure time, the size of the patient or the patient's distance from the PID. The general solution to this is to increase the mAs. If the X-ray appears dark it has an overexposed film. This is fixed by simply reducing the mAs. Figure 6.3 shows the difference between these two errors.

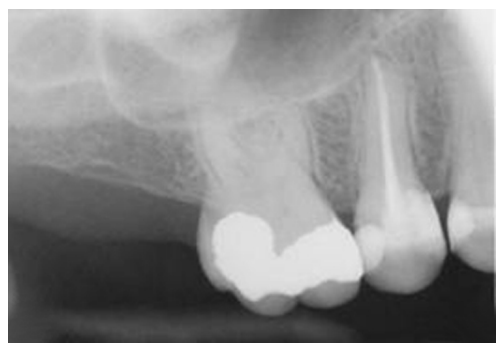

(a)

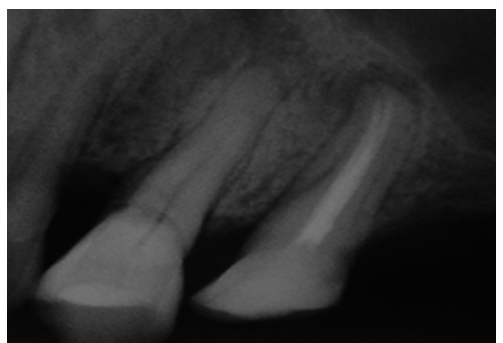

(b)

Figure 6.3: An example of the different exposure rate errors. (a) shows an image with an underexposed film. (b) shows an image with an overexposed film 


\subsubsection{The Dataset}

The data set used for testing was comprised of 115 periodontal X-ray images, 748 pixels in width and 512 pixels in height. The gray levels for each image were on the $[0,255]$ scale. The testing images represented a diverse selection of teeth including molars, canines, incisors and wisdom teeth. In addition to the various errors and artifacts described above, many situations commonly found in dental X-rays were present, such as fillings, missing teeth and impacted teeth. Figure 6.4 highlights some of these different scenarios present in the testing set. Table 6.1 gives a synopsis of the composition of the dataset.

TABLE 6.1: Dataset

\begin{tabular}{lll}
\hline & Upper Jaw & Lower Jaw \\
\hline Total number of images & 37 & 78 \\
Total number of teeth & 141 & 239 \\
PID alignment artifacts & 3 & 20 \\
Underexposed films & 0 & 1 \\
Overexposed films & 3 & 0 \\
Number of fillings & 43 & 57 \\
\hline
\end{tabular}

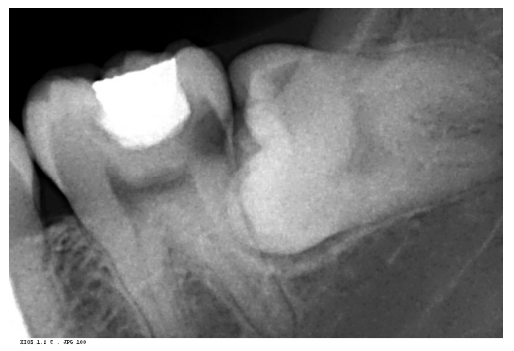

(a)

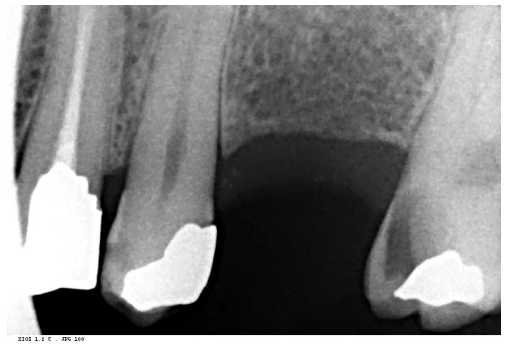

(b)

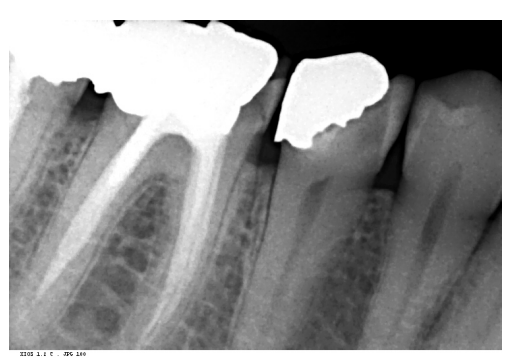

(c)

Figure 6.4: Examples of the different types of X-rays in the dataset. (a). shows an X-ray with an impacted wisdom tooth. (b). shows an X-ray with a missing tooth. (c). shows an X-ray where the contrast darkens across the image. 


\subsection{Caries Detection Framework}

In order to process the dataset, a five stage detection framework was proposed as the best way of handling the X-ray images. The individual stages have been discussed in depth in the previous chapters but the overview is as follows:

- Pre-Processing

- Teeth Segmentation

- Tooth Boundary Detection

- Dental Caries Feature Extraction

- Dental Caries Classification

Figure 6.5 shows an overview of how these steps relate to each other and details the processes involved at each stage of the framework. 


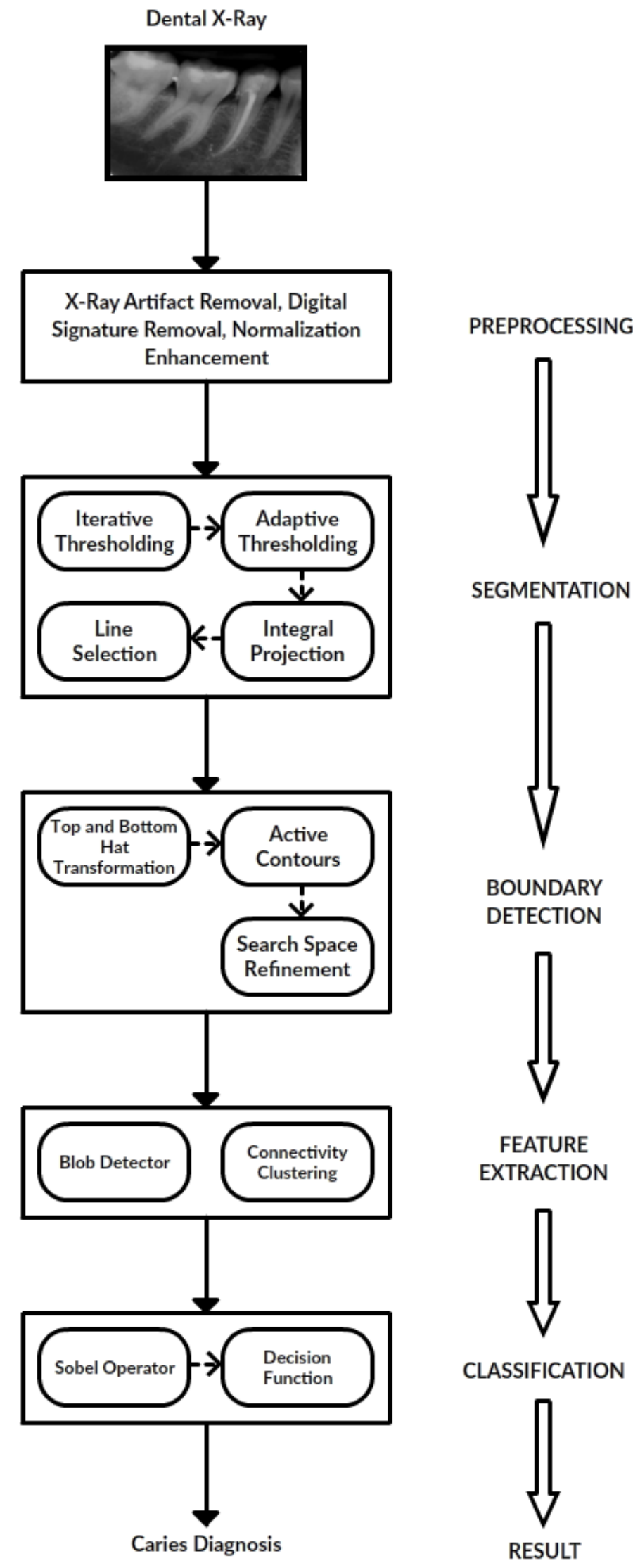

(a)

FIGURE 6.5: Overview of the caries detection framework. 


\subsection{Segmentation Results}

Results relating to the success rate of segmentation methods covered in current research are presented in two ways. The first approach is to separate the performance rates of the upper and lower jaw results and assess them individually. This provides information as to how well the algorithm performed on the different jaw regions. The second approach is that the two regions are grouped, thus using an average of the two rates to represent the algorithms' success as a whole. For the purpose of this dissertation, the results will be compared on an individual jaw region where possible. Table 6.2 shows the results of the segmentation process.

TABLE 6.2: Segmentation Results

\begin{tabular}{lll}
\hline & Upper Jaw & Lower Jaw \\
\hline Total number of teeth in 114 images & 141 & 239 \\
Number of correctly separated teeth & 120 & 216 \\
Correctly separated teeth (\%) & $\mathbf{8 5}$ & $\mathbf{9 0}$ \\
\hline
\end{tabular}

Teeth are considered correctly separated if the separation line did not cause partial separation or division of the teeth. Teeth which were already partial as a result of being at the edge of the X-ray are considered correctly separated if no further partiality was caused. Teeth which were not correctly segmented were either caused as a result of extremely poor contrast, where the enhancement techniques could not establish a distinction between teeth and non-teeth structures, or due to impacted teeth.

A regional comparison of the results against those obtained by Nomir and AbdelMottaleb is presented in Table 6.3. This comparison is made as the algorithms presented in [29] were either wholly or partially adapted for the proposed segmentation method.

Table 6.3: Region Specific Segmentation Results Comparison

\begin{tabular}{lll}
\hline & Upper Jaw & Lower Jaw \\
\hline Oliveira [16] & $72 \%$ & $72 \%$ \\
Nomir and Abdel-Mottaleb [29] & $84 \%$ & $81 \%$ \\
Proposed approach & $\mathbf{8 5 \%}$ & $\mathbf{9 0 \%}$ \\
\hline
\end{tabular}

It should be noted that, with a combination of the adapted and novel algorithms, the segmentation results improved. Table 6.4 provides a comparison of the proposed method to other implementations of the segmentation process described in [28]. 
TABLE 6.4: Overall Segmentation Results Comparison

\begin{tabular}{lll}
\hline & Accuracy (\%) & Implementation \\
\hline Nomir and Abdel-Mottaleb [29] & 82.5 & Thresholding \\
Said et al. [52] & 83 & Thresholding \\
Shah et al. [38] & 58.1 & Active contour \\
Phong-Dinh et al. [28] & 77.23 & Thresholding and integral projection \\
Oliveira [16] & 71.91 & Active contour without edge \\
Lai and Lin [53] & 83 & Region growing \\
Proposed approach & $\mathbf{8 7 . 5}$ & Thresholding and integral projection \\
\hline
\end{tabular}

These results indicate that the method proposed in this dissertation offers a noticeable improvement on existing models. Furthermore, it indicates the diagnostic algorithm received the greatest quantity of correctly segmented images for evaluation. Figure 6.6 shows some examples of the segmentation results.
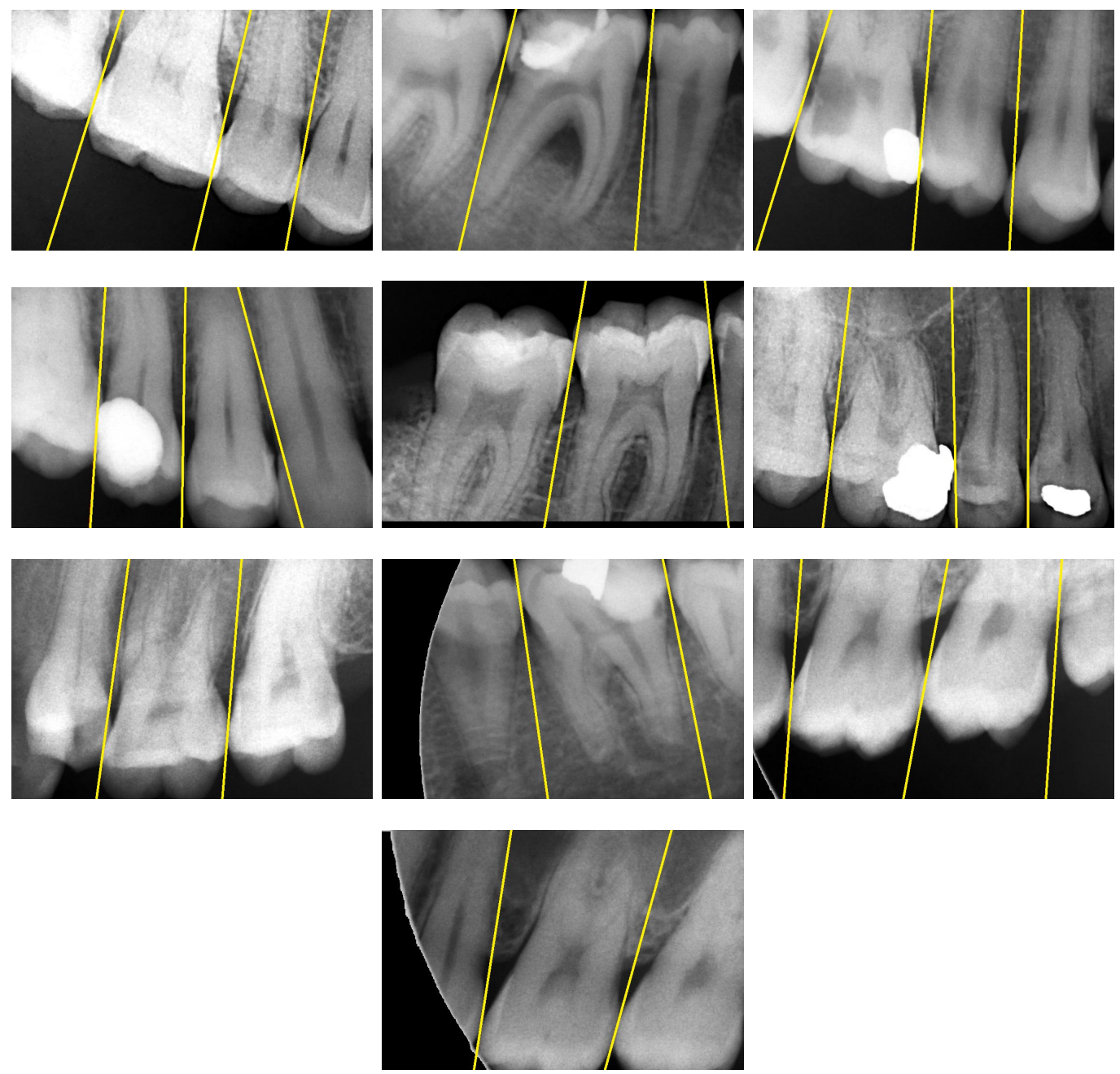

FiguRE 6.6: Examples of segmentation results across the dataset 
TABLE 6.5: Caries Identification Results

\begin{tabular}{lll}
\hline & Upper Jaw & Lower Jaw \\
\hline Total number of caries in the ground truth & 28 & 41 \\
Number of correctly identified teeth & 26 & 29 \\
Correctly identified teeth (\%) & $\mathbf{9 3}$ & $\mathbf{8 1}$ \\
\hline
\end{tabular}

\subsection{Caries Detection Results}

A collection of ground truth data was used to evaluate the success rate of the detection method. The data contained markers for the location of identified caries, as well as the locations of false positive regions. The false positive regions are defined as locations along the boundary of each tooth where caries are incorrectly identified. This occurs due to a misinterpretation of the region, either due to the contrast of the X-ray, or because a partial set of caries identifiers were present which led to the algorithm interpreting the results as a caries location. Table 6.5 shows the caries detection algorithm's success rate of identifying the caries locations in the ground truth, while 6.7 shows some examples of the results obtained by detection algorithm in relation to the ground truth.

TABLE 6.6: Caries Identification Results Breakdown

\begin{tabular}{lll}
\hline & Upper Jaw & Lower Jaw \\
\hline Number of correctly classified teeth & 136 & 220 \\
Number of false positives & 3 & 7 \\
Number of missed caries & 2 & 12 \\
& & \\
Correctly categorized teeth (\%) & 96 & 92 \\
False positives (\%) & 2 & 3 \\
Missed caries (\%) & 2 & 5 \\
\hline
\end{tabular}

The ground truth data did not contain all possible caries locations in the dataset. In order to further investigate the results of the detection algorithm Table 6.6 provides a more in-depth breakdown of the regions identified as caries. Figure 6.7 represents a situation where the ground truth only has false positive markers for teeth where caries are present. 


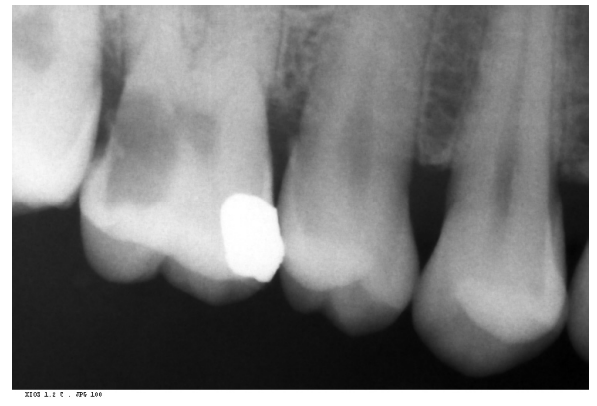

(a)

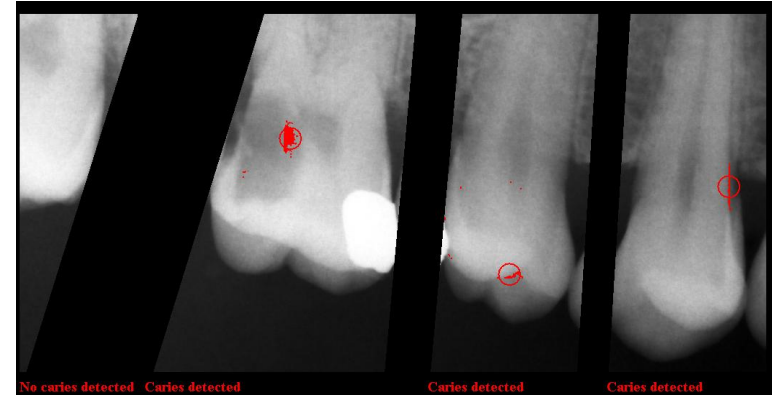

(b)

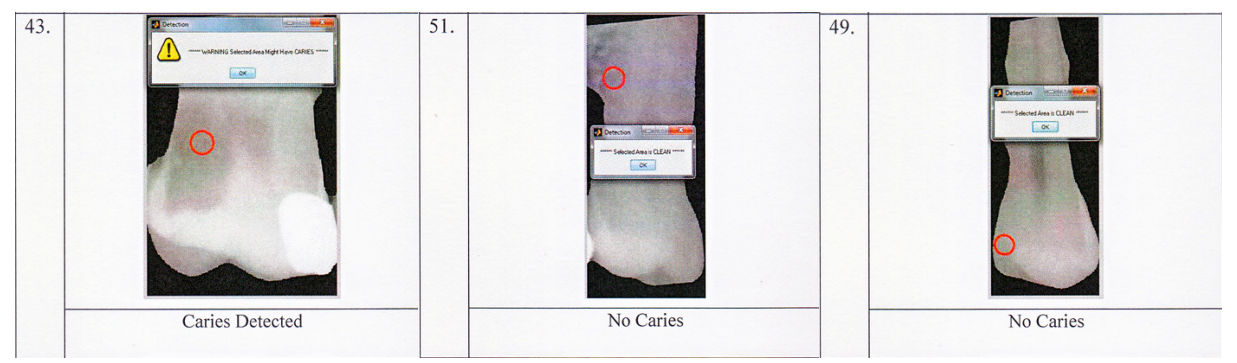

(c)

Figure 6.7: Examples of the results obtained by the detection algorithm and the comparison with the ground truth. (a). the original image. (b). the segmented image with detected caries. (c). the ground truth data

To determine whether these rates fall within acceptable limits, a comparison was done against the different diagnostic methods available. These comprised of caries detection performed by dentists using the Logicon Caries Detector system, as discussed by Tracy et al. [24], unassisted caries diagnosis by dentists, as discussed by Dykstra [25], and caries detection preformed by a supervised learning model, using the method proposed by Oliveira [16]. The results of this comparison can be seen in Table 6.7.

TABLE 6.7: Caries Identification Results Comparison

\begin{tabular}{llll}
\hline & $\begin{array}{l}\text { Correctly } \\
\text { categorized (\%) }\end{array}$ & False positives (\%) & Missed caries (\%) \\
\hline Tracy [24] & 94 & - & 6 \\
Dykstra [25] & 60 & 20 & 20 \\
Oliveira [16] & 98 & - & 2 \\
Valizadeh et al. [54] & 90 & - & 10 \\
Proposed approach 96 & 2 & 2 \\
\hline
\end{tabular}

Despite Oliveira's classification rate being marginally higher than that obtained by the method proposed in this dissertation, the results of the proposed system fall within an 
acceptable range for a novel approach. Furthermore, the diagnostic accuracy of the proposed method achieves better results than existing detection software and performs even better when compared to un-assisted caries diagnosis. The slight loss of performance accuracy can be attributed to the differences in the databases analyzed, where the results in [16] are representative of a detection algorithm being used on a database of panoramic X-rays.

\subsection{Conclusion}

In this chapter the dataset, the caries detection framework and the results obtained from that framework were discussed. Due to the dataset being comprised of non-perfect images, the various nuances associated with the dataset were expanded upon in order to provide a better understanding of the images being processed. In order to understand how all the previous processes combined to reach the final results, the caries detection framework was discussed in its entirety. Once this information had been established, it was possible to present the results obtained from the framework and see how they compared to existing models. The results showed that the proposed framework performed favourably, with results exceeding those of established methods.

The next chapter will look at the implication of the results discussed in this chapter as well as draw more in depth conclusions as to what the results mean. It will also look to future work which can be done to enhance the caries diagnosis framework described in this chapter. 


\section{Chapter 7}

\section{Conclusion and Future Work}

\subsection{Conclusion}

There is a large degree of untapped potential when it comes to CAD systems dealing with dental radiographs. This is especially true for systems focusing on caries detection. Much of the research done with regards to dental radiographs deals with the segmentation of images and to a lesser degree, human recognition through dental profile matching. Due to the reluctance of healthcare professionals to use dental CAD systems because of their high false positive rates, little research has been done into improving existing systems and researching new ones. The problems relating to these existing systems need to be fixed in order to advance this field of research and promote the use of CAD systems as a viable means to treat patients.

Caries detection through implementation of an unsupervised model is one approach to fixing the problems with existing systems. The issue arises due to the way current diagnostic models handle data to reach a diagnosis. The reliance of these systems to determine the presence of caries through use of a database, which is comprised of caries identifier hallmarks, results in systems that are tied to their training data. As a result, should these systems encounter scenarios where partial conditions are met, the diagnostic algorithms err on the side of caution and determine the investigated regions contain caries, often leading to false positives. The use of image analysis techniques to determine the presence of caries aims to create a system that takes a human diagnostic approach, whereby the caries are diagnosed based on visual interpretation of the teeth. 
In this research work, an unsupervised learning model for a caries detection was proposed. The model is implemented using a segmentation method to separate the X-rays into individual teeth, a boundary detection method to determine the edges of the teeth for caries analysis and finally a diagnostic algorithm that assesses the boundary using image analysis techniques.

The segmentation method was implemented via adaptations to existing algorithms in order to obtain the threshold images for integral projection, as well as novel algorithms which were formed by a combination of adapted algorithms. The boundary detection was handled by a combination of top and bottom hat transformations, a binary threshold and an active contour model. Finally, the diagnostic method was handled by blob detection and Sobel kernels.

When the proposed segmentation method was compared against existing models the results proved favourable. The new method showed a $5 \%$ improvement over the original on which it was initially based. Furthermore, it was $4.5 \%$ more accurate than the best method in the comparisons. This is because the proposed method combined aspects of integral projection with rotation with probability functions in order to determine separation lines. This meant that in cases where sub-optimal integral projection lines provided better separation, the weighting component of the probability function ensured the best lines were selected.

The caries detection algorithm was able to obtain favourable results compared to other detection models, with a correct diagnosis rate of $96 \%$. Furthermore, it achieved these results by using a diagnostic model which is less likely to be affected by X-ray variance over a large number of test cases.

It can therefore be concluded that the proposed framework described in this dissertation provides a new approach to both the problems of tooth segmentation and caries detection. The proposed segmentation method offers a more robust method for determining segmentation lines which in turn assists with both supervised and unsupervised learning models as there is a higher likelihood of correctly separated teeth being processed. The success rate of the proposed caries detection algorithm provides a potential avenue for future research using unsupervised models. This will allow for the diagnosis of potential caries to occur without interference from a learning set, thus leading to better results. 


\subsection{Future Work}

The proposed caries detection model uses image analysis to determine the presence of caries in X-ray images and, as such, the final results depend heavily on the quality of the image by the time it reaches the diagnostic method. Optimization of the model at several key processes, including the detection method itself, could improve the final result and future work will look at how to implement these optimizations.

The proposed method is strongly tied to achieving segmentation from a threshold implementation. The dataset that was used was relatively noise free, thus minimal preprocessing needed to be implemented for noise removal. Future work on the model will look at the implementation of additional noise removal filters in order to maintain the accuracy of the segmentation process should noisy images be introduced to the test set.

The second point of optimization is to refine the integral projection method so that only gaps between teeth are considered when creating separation lines. Although algorithms are implemented which determine if a line is attempting to segment the image through a molar tooth, this calculation is only performed after the best line has been determined. Furthermore, in some instances incorrect tooth segmentation occurs when the algorithm does not remove these lines. Future work will look at identifying these gaps as the space between molar roots prior to line generation. This will reduce computation time and improve segmentation results.

The third aspect that can be optimized is the top and bottom hat transformation that is performed in order to implement boundary detection. A single pass transformation leaves some non-pixel elements in the image which needs to be removed with a binary threshold, potentially causing some unwanted image degradation. A two pass transformation results in almost complete pixel degradation of some of the teeth. Future work will look at the possibility of either implementing a half step algorithm to remove the remaining non-tooth pixels, or the implementation of an entirely new method for pixel cleanup.

Finally, the caries detection algorithm uses the predicted size of the tooth to define its analysis space for image analysis. This is dependent on the projected depth of the enamel using average thickness values. As a result, the search regions are not always 
perfectly defined which results in reduced accuracy. Future work will look a new way of determining this search space that is unaffected by estimated sizes and thickness.

In addition to these optimizations, future work will look at expanding the system's ability to diagnose different images. Currently the proposed model has been used to only test bitewing X-rays from the same dataset, however there is scope to expand this. This will initially be achieved by testing new bitewing X-rays from different datasets, however it will eventually be expanded to test a variety of different X-ray types. 


\section{Bibliography}

[1] Human tooth anatomy. URL http://www.mouthhealthy.org/en/az-topics/t/ tooth. [Accessed October 05, 2016].

[2] Theo Vos, Abraham D Flaxman, Mohsen Naghavi, Rafael Lozano, Catherine Michaud, Majid Ezzati, Kenji Shibuya, Joshua A Salomon, Safa Abdalla, Victor Aboyans, et al. Years lived with disability (ylds) for 1160 sequelae of 289 diseases and injuries 1990-2010: a systematic analysis for the global burden of disease study 2010. The Lancet, 380(9859):2163-2196, 2013.

[3] Rafael da Silveira Moreira. Epidemiology of dental caries in the world. INTECH Open Access Publisher, 2012.

[4] Hugh Silk. Diseases of the mouth. Primary Care: Clinics in Office Practice, 41(1): 75-90, 2014.

[5] PJ Holloway and WJ Moore. The role of sugar in the aetiology of dental caries: 1. sugar and the antiquity of dental caries. Journal of dentistry, 11(3):189-190, 1983.

[6] Rickne C Scheid. Woelfel's dental anatomy. Lippincott Williams \& Wilkins, 2012.

[7] Joen I Haring, Joen M Iannucci, and Laura Jansen Lind. Radiographic interpretation for the dental hygienist. WB Saunders Company, 1993.

[8] M Zangooei Booshehry, H Fasihinia, M Khalesi, and L Gholami. Dental caries diagnostic methods. DJH., 2(1), 2011.

[9] Michael G Newman, Henry Takei, Perry R Klokkevold, and Fermin A Carranza. Carranza's clinical periodontology. Elsevier health sciences, 2011.

[10] Noorhayati Mohamed Noor, Noor Elaiza Abdul Khalid, Mohd Hanafi Ali, and Alice Demi Anak Numpang. Fish bone impaction using adaptive histogram equalization 
(ahe). In Computer Research and Development, 2010 Second International Conference On, pages 163-167. IEEE, 2010.

[11] Masahiko Sakata and Koichi Ogawa. Noise reduction and contrast enhancement for small-dose x-ray images in wavelet domain. In 2009 IEEE Nuclear Science Symposium Conference Record (NSS/MIC), pages 2924-2929. IEEE, 2009.

[12] Siti Arpah Ahmad, Mohd Nasir Taib, Noor Elaiza Abdul Khalid, and Haslina Taib. An analysis of image enhancement techniques for dental x-ray image interpretation. International Journal of Machine Learning and Computing, 2(3):292-297, 2012.

[13] J. B. Price. A review of dental caries detection technologies, 2013.

[14] Hiroshi Fujita, Xuejun Zhang, Shoji Kido, Takeshi Hara, Xiangrong Zhou, Yuji Hatanaka, and Rui Xu. An introduction and survey of computer-aided detection/diagnosis (cad). In 2010 international conference on future computer, control and communication (FCCC2010), pages 200-205, 2010.

[15] Bennett T Amaechi. Emerging technologies for diagnosis of dental caries: The road so far. Journal of Applied Physics, 105(10):102047, 2009.

[16] João Oliveira. Caries Detection in Panoramic Dental X-ray Images, 2009.

[17] Hanieh Nokhbatolfoghahaie, Marzieh AliKhasi, Nasim Chiniforush, Farzaneh Khoei, Nassimeh Safavi, and Behnoush Yaghoub Zadeh. Evaluation of accuracy of diagnodent in diagnosis of primary and secondary caries in comparison to conventional methods. Journal of lasers in medical sciences, 4(4):159, 2013.

[18] Grace F Olsen, Susan S Brilliant, David Primeaux, and Kayvan Najarian. An imageprocessing enabled dental caries detection system. In Complex Medical Engineering, 2009. CME. ICME International Conference on, pages 1-8. IEEE, 2009.

[19] Carmine D Clemente. Anatomy. Wolters Kluwer/Lippincott Williams \& Wilkins Health,, 2011.

[20] Anne Waugh and Allison Grant. Ross 8 Wilson anatomy and physiology in health and illness. Elsevier Health Sciences, 2010.

[21] JR Mellberg. Demineralization and remineralization of root surface caries. Gerodontology, 5(1):25-31, 1986. 
[22] G Stookey. Should a dental explorer be used to probe suspected carious lesions? no-use of an explorer can lead to misdiagnosis and disrupt remineralization. Journal of the American Dental Association (1939), 136(11):1527-1529, 2005.

[23] H Fasihinia, M Khalesi, L Gholami, et al. Dental caries diagnostic methods. Avicenna Journal of Dental Research, 2(2):1-12, 2011.

[24] Kyle D Tracy, Bradley A Dykstra, David C Gakenheimer, James P Scheetz, Stephanie Lacina, William C Scarfe, and Allan G Farman. Utility and effectiveness of computer-aided diagnosis of dental caries. General dentistry, 59(2):136-144, 2010.

[25] Bradley Dykstra. Interproximal caries detection: how good are we? Dentistry today, 27(4):144-146, 2008.

[26] Jeong Hyun Yoon and Yong Man Ro. Enhancement of the contrast in mammographic images using the homomorphic filter method. IEICE TRANSACTIONS on Information and Systems, 85(1):298-303, 2002.

[27] K Kavitha Bharathi, S Muruganand, and Azha Periasamy. Digital image processing based noise reduction analysis of digital dental xray image using matlab. Journal of NanoScience and NanoTechnology, 2(1):198-203, 2014.

[28] Abdolvahab Ehsani Rad, Mohd Shafry Mohd Rahim, Amjad Rehman, Ayman Altameem, and Tanzila Saba. Evaluation of current dental radiographs segmentation approaches in computer-aided applications. IETE Technical Review, 30(3):210-222, 2013.

[29] Omaima Nomir and Mohamed Abdel-Mottaleb. A system for human identification from x-ray dental radiographs. Pattern Recognition, 38(8):1295-1305, 2005.

[30] Shiying Hu, Eric A Hoffman, and Joseph M Reinhardt. Automatic lung segmentation for accurate quantitation of volumetric x-ray ct images. IEEE transactions on medical imaging, 20(6):490-498, 2001.

[31] Omaima Nomir and Mohamed Abdel-Mottaleb. Human identification from dental $\mathrm{x}$-ray images based on the shape and appearance of the teeth. IEEE Transactions on information forensics and security, 2(2):188-197, 2007. 
[32] Phen-Lan Lin, Yan-Hao Lai, and Po-Whei Huang. Dental biometrics: Human identification based on teeth and dental works in bitewing radiographs. Pattern Recognition, 45(3):934-946, 2012.

[33] Anil K Jain and Hong Chen. Matching of dental x-ray images for human identification. Pattern recognition, 37(7):1519-1532, 2004.

[34] Dariusz Frejlichowski and Robert Wanat. Automatic segmentation of digital orthopantomograms for forensic human identification. In International Conference on Image Analysis and Processing, pages 294-302. Springer, 2011.

[35] Jindan Zhou and Mohamed Abdel-Mottaleb. A content-based system for human identification based on bitewing dental x-ray images. Pattern Recognition, 38(11): 2132-2142, 2005.

[36] Michael Kass, Andrew Witkin, and Demetri Terzopoulos. Snakes: Active contour models. International journal of computer vision, 1(4):321-331, 1988.

[37] Ashraf A Aly, Safaai Bin Deris, and Nazar Zaki. Research review for digital image segmentation techniques. International Journal of Computer Science $\&$ Information Technology, 3(5):99-106, 2011.

[38] Samir Shah, Ayman Abaza, Arun Ross, and Hany Ammar. Automatic tooth segmentation using active contour without edges. In 2006 Biometrics Symposium: Special Session on Research at the Biometric Consortium Conference, pages 1-6. IEEE, 2006.

[39] Abdolvahab Ehsani Rad, Mohd Shafry Mohd Rahim, and Alireza Norouzi. Digital dental x-ray image segmentation and feature extraction. Indonesian Journal of Electrical Engineering and Computer Science, 11(6):3109-3114, 2013.

[40] A Solanki, K Jain, and N Desai. Isef based identification of ret/filling in dental caries of decayed tooth. International Journal Image Processing (IJIP), 7(2):149$162,2013$.

[41] Stefan Oprea, Costin Marinescu, Ioan Lita, Mariana Jurianu, Daniel Alexandru Visan, and Ion Bogdan Cioc. Image processing techniques used for dental x-ray image analysis. In 2008 31st International Spring Seminar on Electronics Technology, pages 125-129. IEEE, 2008. 
[42] Hui Zhang, Michael J Boyles, Guangchen Ruan, Huian Li, Hongwei Shen, and Masatoshi Ando. Xsede-enabled high-throughput lesion activity assessment. In Proceedings of the Conference on Extreme Science and Engineering Discovery Environment: Gateway to Discovery, page 10. ACM, 2013.

[43] Pedro HM Lira, Gilson A Giraldi, and Luiz AP Neves. Panoramic dental x-ray image segmentation and feature extraction. In Proceedings of $V$ workshop of computing vision, Sao Paulo, Brazil, 2009.

[44] Bernd Fischer, Volker Roth, and Joachim M Buhmann. Clustering with the connectivity kernel. Advances in Neural Information Processing Systems, 16:89-96, 2004.

[45] Toshiaki Kondo, SH Ong, and Kelvin WC Foong. Tooth segmentation of dental study models using range images. IEEE Transactions on medical imaging, 23(3): 350-362, 2004.

[46] Tony Lindeberg. Feature detection with automatic scale selection. International journal of computer vision, 30(2):79-116, 1998.

[47] Stephen J Chu. Range and mean distribution frequency of individual tooth width of the maxillary anterior dentition. Practical Procedures and Aesthetic Dentistry, 19(4):209, 2007.

[48] Robert Jaynes. Caries diagnosis. 2012.

[49] Flávio Vellini-Ferreira, Flávio Augusto Cotrim-Ferreira, José Alaor Ribeiro, and Rívea Inês Ferreira-Santos. Mapping of proximal enamel thickness in permanent teeth. Brazilian Journal of Oral Sciences, 11(4):481-485, 2012.

[50] Hanno Scharr. Optimal operators in digital image processing. $\mathrm{PhD}$ thesis, 2000.

[51] Universiti Teknologi Malaysia Dental Clinic, Health Center. Dental x-ray (periapical) dataset, 2013.

[52] Eyad Haj Said, Diaa Eldin M Nassar, Gamal Fahmy, and Hany H Ammar. Teeth segmentation in digitized dental x-ray films using mathematical morphology. IEEE transactions on information forensics and security, 1(2):178-189, 2006. 
[53] YH Lai and PL Lin. Effective segmentation for dental x-ray images using texturebased fuzzy inference system. In International Conference on Advanced Concepts for Intelligent Vision Systems, pages 936-947. Springer, 2008.

[54] Solmaz Valizadeh, Mostafa Goodini, Sara Ehsani, Hadis Mohseni, Fateme Azimi, and Hooman Bakhshandeh. Designing of a computer software for detection of approximal caries in posterior teeth. Iranian Journal of Radiology, 12(4), 2015. 